\title{
SYSTEMIC REVIEWS / META-ANALYSIS
}

\section{Volunteer Impact on Health-Related Outcomes for Seniors: a Systematic Review And Meta-Analysis}

Ainsley Moore, MD, CFPC, MSc${ }^{1}$, Shahrzad Motagh, DVM, MSc $^{2}$, Behnam Sadeghirad, MSc, PhD $^{2}$, Housne Begum, MSc, $\mathrm{PhD}^{1}$, John J. Riva, DC, MSc, PhD ${ }^{1,3,4}$, Jessica Gaber, Mws, RSw ${ }^{1}$, Lisa Dolovich, BScPhm, PharmD, $\mathrm{msc}^{1,3,4}$

${ }^{1}$ Department of Family Medicine, McMaster University, Hamilton, ON; ${ }^{2}$ The Michael G. DeGroote Institute of Pain Research and Care, McMaster University, Hamilton, ON: ${ }^{3}$ Health Evidence and Impact, McMaster University, Hamilton, ON; ${ }^{4}$ Leslie Dan Faculty of Pharmacy, University of Toronto, Toronto, ON, Canada

https://doi.org/10.5770/cgj.24.434

\section{ABSTRACT}

\section{Background}

Volunteers are increasingly promoted to improve healthrelated outcomes for community-dwelling elderly without synthesized evidence for effectiveness. This systematic review and meta-analysis evaluates the effects of unpaid volunteer interventions on health-related outcomes for such seniors.

\section{Methods}

MEDLINE, EMBASE and Cochrane (CENTRAL) were searched up to November 2018. We included English language, randomized trials. Two reviewers independently identified studies, extracted data, and assessed evidence certainty (using GRADE). Meta-analysis used random-effects models. Univariate meta-regressions investigated the relationship between volunteer intervention effects and trial participant age, percentage females, and risk of bias.

\section{Results}

28 included studies focussed on seniors with a variety of chronic conditions (e.g., dementia, diabetes) and health states (e.g., frail, palliative). Volunteers provided a range of roles (e.g., counsellors, educators and coaches). Low certainty evidence found that volunteers may improve both physical function $(\mathrm{MD}=3.2$ points on the 100-point SF-36 physical component score [PCS]; $95 \%$ CI: $1.09,5.27)$ and physical activity levels $(\mathrm{SMD}=0.5$, $95 \%$ CI: 0.14 to 0.83 ). Adverse events were not increased.

\section{Conclusion}

Volunteers may increase physical activity levels and subjective ratings of physical function for seniors without apparent harm. These findings support the WHO call to action on evidence-based policies to align health systems in support of older adults.

Key Words: volunteers, geriatrics, community

\section{INTRODUCTION}

Increasing longevity is a main driver of population aging worldwide. This trend has major implications for the healthcare sector, particularly human health resources where traditional family structures have changed dramatically with increased numbers of older people living alone and without informal caregivers. ${ }^{(1)}$ The world's population aged 60 years and older will double and total about two billion by 2050 , and is expected to carry the highest burden of chronic disease, requiring increasingly complex care management across multiple sectors of primary, community, and home-based care. ${ }^{(2)}$

As community builders and cultivators of social connectedness, volunteers are needed and increasingly recruited and trained to work within the health-care system to add relational support, improve connection with social and health-care systems, and augment health professional resources and services. ${ }^{(3)}$ New programs (e.g., home-based care and treatment support) emphasizing the volunteer role in the community to improve health-care integration and health outcomes for older adults are emerging; ${ }^{(4-7)}$ however, this entire body of evidence has not been synthesized to date.

Previous reviews have summarized separately some aspects of volunteer impact for those living with cancer, depression, and diabetes among children and general adult populations (not specifically adults over 55 years of age)..$^{8-}$ 10) Closer to our population of interest, a narrative subgroup analysis from a Cochrane review found that community lay health workers improved subjective well-being, happiness, physical health, and contentment, with no improvement in mortality, disability, or mental health status for the elderly ${ }^{(11)}$ (although these interventions included paid lay health workers).

Based on the overarching hypothesis from the Health TAPESTRY ${ }^{(12)}$ study, we proposed (aprior) that community volunteer activity, comparable to community or lay health worker activities, would improve patient-reported outcomes for older adults. To that end, we reviewed the literature in 
older populations to assess the impact specifically of unpaid volunteers on physical activity, self-reported mental and physical health, quality of life, falls, hospitalization, and harms (adverse events) experienced by elderly persons residing in the community. Our broad outcomes of interest (physical health and mental well-being) were not only limited to recipients of volunteer care and could include such impacts on volunteers who may themselves be older adults. It is important to acknowledge that, in addition to our outcomes of interest, there are deep and meaningful social gains (e.g., companionship, camaraderie, self-esteem) that are experienced particularly by older volunteers, as well as instrumental benefits of volunteering (e.g., skills development and employability) as experienced by younger volunteers. ${ }^{(13)}$

Aligned with the WHO strategy on aging to "maintain functional ability and well-being in older age", ${ }^{(1)}$ this paper aims to support the development of evidence-informed policy for clinical leaders, health system planners, volunteer organizations, and citizens regarding health and social system planning for the increasing populations of seniors residing in the community.

\section{METHODS}

\section{Protocol Registration}

A protocol for this review was registered with PROSPERO (CRD42019116541).

\section{Data Sources}

We searched MEDLINE, EMBASE and the Cochrane Central Register of Controlled Trials (CENTRAL) for relevant published randomized control trials (RCTs), from database inception to November 2018, without language restriction. Appendix A provides the search strategy. We also searched the reference lists of included studies and relevant reviews for additional eligible trials.

\section{Study Selection}

Reviewers screened the titles and abstracts of all identified studies, independently and in duplicate, using a priori selection criteria. Subsequently, reviewers assessed full texts for potentially eligible studies, and resolved disagreements through consensus or third-party consultation with the authorship team. We screened for inclusion, English language trials that randomized adults residing in the community aged 55 years or older to volunteer interventions or to usual/standard of care, waitlist control, or no intervention. To be included, volunteers were the primary intervention. We excluded studies where participants were hospitalized or resided in institutional settings (e.g., hospitals, long-term care, prison) or workplace settings. We also excluded studies that compensated volunteers (paid volunteer personnel) or did not report any of our outcomes of interest.

Our outcomes of interest were: subjective reports from older adults of physical health (physical function, physical activity levels), mental health (emotional function, anxiety, depression), and quality of life, as well as objective outcomes of frequency of falls, hospital admissions, and lastly, adverse events associated with volunteer interventions. Specifically, we were interested in the following outcomes, as measured by these six metrics:

\section{Physical Health}

a) Physical functioning as reported using the 'physical functioning' domains from validated tools such as: the 36-Item and 12-Item Short Form Health Surveys (SF-36, SF-12), ${ }^{(14)}$ the Western Ontario and McMaster Universities Osteoarthritis Index (WOMAC), ${ }^{(15)}$ and the WHO Quality of Life-BREF (WHOQOL-BREF). ${ }^{(16)}$ If the 'physical functioning' domain was not available, the physical component summary scores of the SF-36 or SF-12 (which incorporate the domains of physical functioning and role physical) were used. ${ }^{(14)}$

b) Physical activity as reported using validated metrics such as: time spent in moderate to vigorous physical activity (MVPA) ${ }^{(17)}$ (e.g., minutes of exercise per week) or the metabolic equivalent of task (MET) (e.g., energy spent in activity per kg of weight). ${ }^{(18)}$

\section{Mental Health}

a) Emotional functioning as reported using the 'emotional functioning' domain from validated tools such as the SF-36 and SF-12(14) or the WHOQOL-BREF. ${ }^{(16)}$ If the 'emotional functioning' domain was not available, the mental component summary scores (which include emotional functioning) from the SF-36 or SF-12 ${ }^{(14)}$ were used.

b) Anxiety as reported using the anxiety subscales of the Hospital Anxiety and Depression Scale (HADS), ${ }^{(19)}$ the Mental Health Inventory $(\mathrm{MHI}),{ }^{(20)}$ or other validated tools.

c) Depression as reported using the depression subscales of the HADS, ${ }^{(19)}$ the MHI, the Centre for Epidemiological StudiesDepression (CES-D) scale, ${ }^{(21)}$ the Geriatric Depression Scale, or other validated tools were used. ${ }^{(22)}$

\section{Quality of Life}

Quality of life as reported using the EuroQol-5D (EQ-5D), ${ }^{(23)}$ WHOQOL-BREF, ${ }^{(16)}$ or other validated tools were used.

\section{Falls (as reported)}

\section{Hospitalizations (as reported) \\ Adverse Events (as reported by study authors)}

\section{Data Extraction and Risk of Bias Assessment}

Two reviewers (BS and SM) extracted the following data, independently and in duplicate: general study information (first author's name, publication year, and trial design), participants' details (sample size, age, number of male/female participants, participants' health status/clinical conditions), details on the intervention and comparison (characteristics of volunteers, setting), and outcomes as listed above (Appendix B). In three- or four-arm randomized trials with two active arms, if both interventions were delivered by unpaid volunteers but 
with different intensity or duration, we combined outcome data using methods suggested by the Cochrane Handbook for Systematic Reviews of Interventions. ${ }^{(24)}$ When studies reported their results in multiple follow ups, we extracted data from the longest follow-up time.

The same two reviewers independently assessed risk of bias using a modified Cochrane risk of bias instrument for RCTs that addressed the following issues: random sequence generation, allocation concealment, blinding of study participants, health-care providers, and outcome assessors, incomplete outcome data ( $>20 \%$ missing participant data), and other potential sources of bias. ${ }^{(25,26)}$

\section{Certainty of Evidence Assessment}

To assess the certainty of evidence, we used the Grading of Recommendations, Assessment, Development, and Evaluation (GRADE) approach that classifies evidence as high, moderate, low, or very low certainty on the basis of considerations of risk of bias, inconsistency, indirectness, imprecision, and publication bias. ${ }^{(27)}$ We resolved disagreements between reviewers in data extraction, risk of bias assessments, and assessments of evidence certainty by consensus. We used the MAGIC Authors Publishing Platform (https://app.magicapp. org) to generate the GRADE summary of findings table.

\section{Data Synthesis and Statistical Methods}

Continuous measures were converted to common scales on a domain-by-domain basis as follows: ${ }^{(28)}$

1. Physical functioning was converted to the 100-point SF36 physical component score.

2. Emotional functioning was converted to the 100-point SF-36 mental component score.

3. Quality of life was converted to $0-1$ point EQ-5D score.

4. Depression and anxiety were converted to 10-point HADS (range from 11-21).

For the above-mentioned outcomes, we calculated the mean difference (MD) and its corresponding 95\% confidence interval (CI). The median of the control group of included trials was used as the baseline risk. Due to the nature of measurements for physical activity, we decided to use standardized mean difference (SMD) for pooling these results due to various reporting metrics (e.g., MET, MVPA). For frequency of falls and hospitalizations, we used narrative description to summarize these results, as quantitative pooling was not feasible due to variation in reporting (e.g., total number of individuals experiencing an event and number of falls events). For cluster randomized trials, we used the method suggested by the Cochrane Handbook for Systematic Reviews of Interventions to calculate effective sample size, using the intracluster correlation coefficient or variance inflation factor reported in the original trial. ${ }^{(24)}$

Statistical heterogeneity was assessed using the Q statistic and I2. We used the DerSimonian-Laird random-effects model for the meta-analysis of all outcomes. ${ }^{(24)}$ We performed subgroup analysis for risk of bias on an item-by-item basis. Subgroup analyses were performed when two or more studies were in a given subgroup. We conducted tests of interaction to establish whether the subgroups differed significantly from each other. ${ }^{(29)}$ We performed univariate meta-regressions to assess the effects of participant's age and percentage of female participants on the intervention effects. We examined publication bias using funnel plots for outcomes when 10 or more studies were available. ${ }^{(30)}$ We used Stata software (Version 15.1, StataCorp LLC, College Station, TX, USA) for all statistical analysis.

\section{RESULTS}

\section{Description of Included Studies}

We identified 3,794 titles and abstracts from our searches, of which 139 were deemed eligible for full-text evaluation. Figure 1 provides the details of study selection. We included 27 trials in 28 reports that proved eligible, enrolling 146,937 individuals. The median age of study participants among the included studies was 66.4 years (interquartile range [IQR]: 61.4 to 76.1 ), on average $65.0 \%$ of trial participants were female (IQR: $52.2 \%$ to $82.7 \%$ ). One study took place in a lower-middle income country (Philippines), the remainder took place in high income countries: the USA (9 studies), followed by the United Kingdom (5 studies), Canada and Austria (3 studies each), Australia and Hong Kong (2 studies each), and one study from each of Scotland, Finland, and Argentina (Table 1).

Studies focused on seniors living with a variety of conditions and health states, including: cardiovascular disease, ${ }^{(31-33)}$ osteoarthritis, ${ }^{(34-36)}$ diabetes, ${ }^{(37-39)}$ cancer, ${ }^{(40-44)}$ inactivity, ${ }^{(45-47)}$ dementia, ${ }^{(48)}$ depression, ${ }^{(49)}$ frailty, ${ }^{(50,51)}$ end of life status, ${ }^{(52)}$ and healthy seniors ${ }^{(35,53-56)}$ (Table 1).

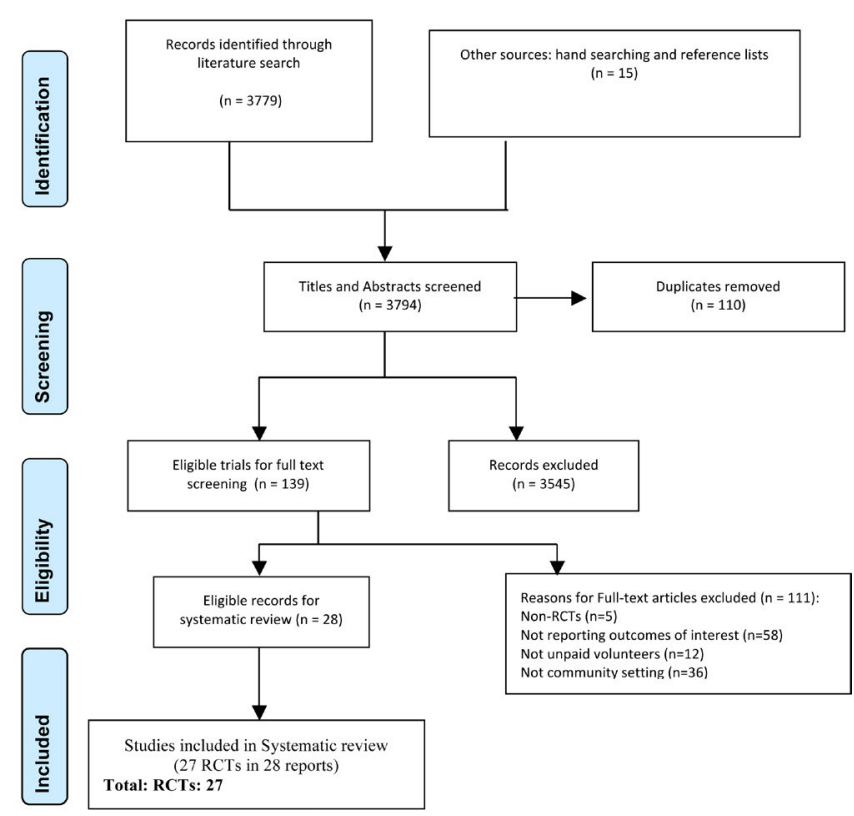

FIGURE 1. PRISMA flow diagram for study selection 
MOORE: VOLUNTEER IMPACT ON SENIORS

TABLE 1

Characteristics of Studies

\begin{tabular}{|c|c|c|c|c|c|c|c|}
\hline $\begin{array}{l}\text { Author } \\
\text { Country } \\
\text { (Year) }\end{array}$ & $\begin{array}{c}\text { Trial } \\
\text { Design }\end{array}$ & $\begin{array}{l}\text { Mean } \\
\text { Age }\end{array}$ & $\begin{array}{c}\% \\
\text { Female }\end{array}$ & $\begin{array}{l}\text { Clinical } \\
\text { Condition }\end{array}$ & Intervention (Details) & $\begin{array}{c}\text { Comparison } \\
\text { (Details) }\end{array}$ & Outcome \\
\hline $\begin{array}{l}\text { Allen }^{(52)} \\
\text { USA } \\
(2014)\end{array}$ & Parallel & 78.2 & 82.0 & Palliative & $\begin{array}{l}\text { Reminiscence }+ \\
\text { creative activity } \\
\text { sessions by retired } \\
\text { seniors }\end{array}$ & $\begin{array}{l}3 \text { Supportive } \\
\text { telephone contacts } \\
\text { by research staff } \\
\text { (no details) }\end{array}$ & Depression \\
\hline $\begin{array}{l}\text { Barlow }^{(31)} \\
\text { UK } \\
(2009)\end{array}$ & Parallel & 66.1 & 27.8 & $\begin{array}{c}\text { Myocardial } \\
\text { infarction }(\mathrm{MI}) \\
\text { patients who } \\
\text { completed cardiac } \\
\text { rehab }\end{array}$ & $\begin{array}{l}\text { Chronic disease self- } \\
\text { management (Expert } \\
\text { Patient Program) by } \\
\text { lay tutors who had } \\
\text { an MI }\end{array}$ & Wait list control & $\begin{array}{c}\text { Physical function, } \\
\text { Physical Activity, } \\
\text { Anxiety, } \\
\text { Depression }\end{array}$ \\
\hline $\begin{array}{l}\text { Buman }^{(45)} \\
\text { USA } \\
(2011)\end{array}$ & Parallel & 63.4 & 82.7 & $\begin{array}{c}\text { Currently } \\
\text { inactive or } \\
\text { insufficiently } \\
\text { active }\end{array}$ & $\begin{array}{l}\text { Self-management: } \\
\text { physical activity. } \\
\text { Group sessions by } \\
\text { 'peer mentors' }\end{array}$ & $\begin{array}{l}\text { Standard community- } \\
\text { based physical } \\
\text { activity promotion }\end{array}$ & Physical Activity \\
\hline $\begin{array}{l}\text { Castro }^{(46)} \\
\text { USA } \\
(2011)\end{array}$ & Parallel & 59.1 & 65.8 & $\begin{array}{l}\text { Under-active } \\
\text { healthy elderly }\end{array}$ & $\begin{array}{l}\text { Telephone-based } \\
\text { physical activity } \\
\text { advice delivered } \\
\text { by trained } \\
\text { 'volunteer peers' }\end{array}$ & $\begin{array}{l}\text { Attention-control arm } \\
\text { by staff: telephone } \\
\text { advice heart health } \\
\text { nutrition }\end{array}$ & $\begin{array}{l}\text { Physical Activity } \\
\text { Adverse Events }\end{array}$ \\
\hline $\begin{array}{l}\text { Chan }^{(47)} \\
\text { Hong Kong } \\
(2017)\end{array}$ & Parallel & 77.3 & 76.1 & $\begin{array}{c}\text { Currently inactive } \\
\text { or insufficiently } \\
\text { active }\end{array}$ & $\begin{array}{l}\text { Tai chi qigong } \\
\text { sessions by 'senior } \\
\text { volunteers' }\end{array}$ & $\begin{array}{l}\text { Usual care (irregular } \\
\text { home visits by social } \\
\text { workers) }\end{array}$ & $\begin{array}{l}\text { Physical } \\
\text { Function, } \\
\text { Anxiety } \\
\text { Depression }\end{array}$ \\
\hline $\begin{array}{l}\text { Charlesworth }^{(48)} \\
\text { UK } \\
(2016)\end{array}$ & Factorial & 66.7 & 68.2 & $\begin{array}{l}\text { Patients with } \\
\text { dementia living } \\
\text { at home }\end{array}$ & $\begin{array}{l}\text { 1) Carer Support } \\
\text { Program (CSP) } \\
\text { 2) Remembering } \\
\text { Yesterday Caring } \\
\text { Today (RYCT) } \\
\text { 3) CSP+RYCT by } \\
\text { peer befrienders }\end{array}$ & $\begin{array}{l}\text { Usual Care } \\
\text { (diagnostic } \\
\text { memory and } \\
\text { challenging behaviour } \\
\text { clinics) }\end{array}$ & $\begin{array}{c}\text { Anxiety } \\
\text { Depression } \\
\text { Quality of Life } \\
\text { Adverse Events/ } \\
\text { Harms }\end{array}$ \\
\hline $\begin{array}{l}\text { Coull(32) } \\
\text { Scotland } \\
(2004)\end{array}$ & Parallel & 67.5 & 39.5 & $\begin{array}{c}\text { Inpatients \& } \\
\text { outpatients } \\
\text { attending } \\
\text { secondary care } \\
\text { with a diagnosis } \\
\text { of angina or } \\
\text { acute MI }\end{array}$ & $\begin{array}{l}\text { Cardiovascular } \\
\text { disease self- } \\
\text { management } \\
\text { Group sessions led } \\
\text { by lay mentors }\end{array}$ & Standard Care & $\begin{array}{c}\text { Physical Function, } \\
\text { Depression, Anxiety } \\
\text { Adverse Events/ } \\
\text { Harms }\end{array}$ \\
\hline $\begin{array}{l}\text { Crane-Okada }{ }^{(40)} \\
\text { USA } \\
(2014)\end{array}$ & Parallel & 61.8 & 100.0 & $\begin{array}{l}\text { Post-operative } \\
\text { breast cancer } \\
\text { surgery }\end{array}$ & $\begin{array}{l}\text { Telephone-based } \\
\text { psychosocial support } \\
\text { by 'senior peer } \\
\text { counsellors' }\end{array}$ & No peer contact & Anxiety \\
\hline $\begin{array}{l}\text { Crotty }^{(34)} \\
\text { Australia } \\
(2009)\end{array}$ & Parallel & 67.5 & 60.5 & $\begin{array}{c}\text { Outpatients } \\
\text { awaiting hip or } \\
\text { knee replacement } \\
\text { surgery }\end{array}$ & $\begin{array}{l}\text { Osteoarthritis self- } \\
\text { management course } \\
\text { or individual phone } \\
\text { support by peer } \\
\text { support educators }\end{array}$ & $\begin{array}{l}\text { Usual care } \\
\text { (orthopedic wait list) }\end{array}$ & $\begin{array}{l}\text { Physical Function } \\
\text { Depression } \\
\text { Quality of Life } \\
\text { Adverse Events/ } \\
\text { Harms }\end{array}$ \\
\hline $\begin{array}{l}\text { DeMello }^{(41)} \\
\text { USA } \\
(2008)\end{array}$ & Parallel & 55.6 & 100.0 & $\begin{array}{l}\text { Breast cancer } \\
\text { survivors }\end{array}$ & $\begin{array}{l}\text { Pedometer, heart rate } \\
\text { monitor, telephone } \\
\text { counselling to adopt } \\
\text { physical activity by } \\
\text { volunteer coaches }\end{array}$ & $\begin{array}{l}\text { Information (breast } \\
\text { cancer recovery) } \\
\text { coaches answered } \\
\text { questions by phone }\end{array}$ & $\begin{array}{l}\text { Physical Activity, } \\
\text { Physical Function, } \\
\text { Emotional Function }\end{array}$ \\
\hline
\end{tabular}


MOORE: VOLUNTEER IMPACT ON SENIORS

TABLE 1. Continued

\begin{tabular}{lccccccc}
\hline $\begin{array}{l}\text { Author } \\
\text { Country }\end{array}$ & Trial & Mean & $\%$ & Clinical & Intervention (Details) & Comparison \\
(Year) & Design & Age & Female & Condition & & Outcome \\
\end{tabular}

\begin{tabular}{|c|c|c|c|c|}
\hline $\begin{array}{l}\text { Escolar }^{(53)} \\
\text { Philippines } \\
(2014)\end{array}$ & Parallel & & 60.0 & Healthy elderly \\
\hline $\begin{array}{l}\text { Gagliardino }^{(37)} \\
\text { Argentina } \\
(2013)\end{array}$ & Parallel & 60.9 & 51.5 & Diabetic patients \\
\hline $\begin{array}{l}\text { Haider }^{(50)} \\
\text { Austria } \\
(2018)\end{array}$ & Parallel & 82.8 & 83.8 & $\begin{array}{l}\text { Prefrail and frail } \\
\text { older adults }\end{array}$ \\
\hline $\begin{array}{l}\text { Hind }^{(57)} \\
\text { UK }\end{array}$ & Parallel & 80.9 & 58.6 & $\begin{array}{l}\text { Independently } \\
\text { living elderly }\end{array}$ \\
\hline
\end{tabular}

Third Age Learning No exposure to Depression

Program (wellness, intervention

physical fitness,

and livelihood

training by volunteer

university faculty)

Peer diabetic

educators (group

Professional diabetic

Hospital

sessions)

Admissions

Home based physical Social home visits

(not pooled)

training, nutritional (lay volunteers)

and social support

Physical

by lay volunteers

Performance

(buddies)

Battery

(not pooled)

Individual and group Usual health and Physical Function

phone calls to support social care provision Emotional Function

social connection by

Depression

befrienders

Quality of Life

Adverse Events/

Harms

\begin{tabular}{|c|c|c|c|c|c|c|c|}
\hline $\begin{array}{l}\text { Iliffe }^{(54)} \\
\text { UK } \\
(2014)\end{array}$ & cluster & 71.9 & 63.0 & Healthy elderly & $\begin{array}{l}\text { Class-based or } \\
\text { home-based exercise } \\
\text { program by } \\
\text { peer mentors }\end{array}$ & Usual primary care & $\begin{array}{l}\text { Physical Function } \\
\text { Quality of Life } \\
\text { Falls } \\
\text { Adverse Events/ } \\
\text { Harms }\end{array}$ \\
\hline $\begin{array}{l}\text { Johansson }^{(38)} \\
\text { Austria } \\
(2016)\end{array}$ & cluster & 63.0 & 51.3 & Diabetic patients & $\begin{array}{l}\text { Physical activity } \\
\text { sessions \& diabetes } \\
\text { self-management } \\
\text { groups by } \\
\text { peer supporters }\end{array}$ & $\begin{array}{l}\text { Usual primary care } \\
\text { practices }\end{array}$ & Quality of Life \\
\hline $\begin{array}{l}\text { Kaczorowski }{ }^{(58)} \\
\text { Canada } \\
(2011)\end{array}$ & cluster & 74.8 & 52.2 & $\begin{array}{c}\text { Community } \\
\text { dwelling residents } \\
>65 \text { years of age }\end{array}$ & $\begin{array}{l}\text { Cardiovascular risk } \\
\text { assessment and } \\
\text { education sessions by } \\
\text { peer health educators }\end{array}$ & $\begin{array}{l}\text { Communities } \\
\text { not exposed to } \\
\text { intervention }\end{array}$ & $\begin{array}{l}\text { Hospital } \\
\text { Admissions } \\
\text { (not pooled) }\end{array}$ \\
\hline $\begin{array}{l}\text { Kapan } \\
\text { Austria } \\
(2017)\end{array}$ & Parallel & 82.6 & 83.8 & $\begin{array}{l}\text { Prefrail or frail } \\
\text { elderly }\end{array}$ & $\begin{array}{l}\text { Home based physical } \\
\text { training \& nutritional } \\
\text { advice by lay } \\
\text { volunteers (buddies) }\end{array}$ & $\begin{array}{l}\text { Social home visits } \\
\text { (lay volunteers) }\end{array}$ & $\begin{array}{c}\text { Physical Function } \\
\text { Physical activity } \\
\text { Falls } \\
\text { Quality of Life }\end{array}$ \\
\hline $\begin{array}{l}\text { Leone }^{(42)} \\
\text { USA } \\
(2016)\end{array}$ & Cluster & 62.8 & 68.6 & $\begin{array}{l}\text { Older African } \\
\text { Americans } \\
\text { (average risk for } \\
\text { colon cancer) }\end{array}$ & $\begin{array}{l}\text { Telephone calls to } \\
\text { motivate physical } \\
\text { activity and adhere } \\
\text { to colon cancer } \\
\text { screening by } \\
\text { Church-based peer } \\
\text { counsellors }\end{array}$ & $\begin{array}{l}\text { Comparison churches } \\
\text { (Newsletters } \\
\text { promoting fruit } \\
\text { and vegetable } \\
\text { consumption) }\end{array}$ & Physical Activity \\
\hline $\begin{array}{l}\text { McNeil(49) } \\
\text { Canada } \\
(1995)\end{array}$ & Parallel & 72.5 & 86.7 & $\begin{array}{l}\text { Community- } \\
\text { dwelling, } \\
\text { unhealthy and } \\
\text { unhappy elderly }\end{array}$ & $\begin{array}{l}\text { Home visits } \\
\text { (conversations and or } \\
\text { walking activities } \\
\text { (psychology student } \\
\text { volunteers) }\end{array}$ & Wait list control & $\begin{array}{l}\text { Subjective Physical } \\
\text { Health score (1 item) } \\
\text { Happiness scale } \\
\text { (Not pooled) }\end{array}$ \\
\hline
\end{tabular}


MOORE: VOLUNTEER IMPACT ON SENIORS

TABLE 1. Continued

\begin{tabular}{|c|c|c|c|c|c|c|c|}
\hline $\begin{array}{l}\text { Author } \\
\text { Country } \\
\text { (Year) }\end{array}$ & $\begin{array}{c}\text { Trial } \\
\text { Design }\end{array}$ & $\begin{array}{c}\text { Mean } \\
\text { Age }\end{array}$ & $\begin{array}{c}\% \\
\text { Female }\end{array}$ & $\begin{array}{l}\text { Clinical } \\
\text { Condition }\end{array}$ & Intervention (Details) & $\begin{array}{l}\text { Comparison } \\
\text { (Details) }\end{array}$ & Outcome \\
\hline $\begin{array}{l}\text { Mountain }(55,57) \\
\text { UK } \\
(2014)\end{array}$ & Parallel & 81.0 & 58.6 & $\begin{array}{l}\text { Elderly living } \\
\text { independently }\end{array}$ & $\begin{array}{l}\text { Individual and } \\
\text { group phone calls } \\
\text { to promote social } \\
\text { connection by } \\
\text { befrienders }\end{array}$ & $\begin{array}{l}\text { Usual health and } \\
\text { social care provision }\end{array}$ & $\begin{array}{c}\text { Physical Function } \\
\text { Emotional Function } \\
\text { Quality of Life }\end{array}$ \\
\hline $\begin{array}{l}\text { Parry } \\
\text { Canada } \\
(2009)\end{array}$ & Parallel & 63.0 & 16.8 & $\begin{array}{c}\text { First-time } \\
\text { nonemergency } \\
\text { coronary artery } \\
\text { bypass graft } \\
\text { (CABG) surgery } \\
\text { patients }\end{array}$ & $\begin{array}{l}\text { Individualized } \\
\text { education \& support } \\
\text { via telephone by } \\
\text { cardiac surgery peers }\end{array}$ & $\begin{array}{l}\text { Usual care ('standard' } \\
\text { pre and post CABG } \\
\text { education) }\end{array}$ & $\begin{array}{l}\text { Physical Function } \\
\text { Emotional Function }\end{array}$ \\
\hline $\begin{array}{l}\text { Rantanen }^{(35)} \\
\text { Finland } \\
(2015)\end{array}$ & Parallel & 81.9 & 90.1 & $\begin{array}{l}\text { Elderly with } \\
\text { severe mobility } \\
\text { limitations } \\
\text { (otherwise } \\
\text { healthy) }\end{array}$ & $\begin{array}{l}\text { Out of home activities } \\
\text { (walking, cultural } \\
\text { events, daily errands) } \\
\text { by retired volunteers }\end{array}$ & Waitlist control & $\begin{array}{l}\text { Physical Function } \\
\text { Quality of Life } \\
\text { Adverse Events/ } \\
\text { Harms }\end{array}$ \\
\hline $\begin{array}{l}\text { Robinson }^{(36)} \\
\text { USA } \\
(2006)\end{array}$ & Parallel & 58.6 & 100.0 & $\begin{array}{l}\text { Middle-aged and } \\
\text { older women with } \\
\text { chronic physical } \\
\text { disabilities }\end{array}$ & $\begin{array}{l}\text { Workshops health- } \\
\text { related goal setting, } \\
\text { social connection by } \\
\text { peer supporters }\end{array}$ & Waitlist control & Physical Function \\
\hline $\begin{array}{l}\text { Safford } \\
\text { USA } \\
(2015)\end{array}$ & Cluster & 60.2 & 75.3 & $\begin{array}{l}\text { Diabetic patients } \\
\text { selected for } \\
\text { interest in self- } \\
\text { management }\end{array}$ & $\begin{array}{l}\text { One-to-one planning } \\
\text { for diabetic primary } \\
\text { care visits by 'peer } \\
\text { coaches' }\end{array}$ & $\begin{array}{l}\text { Group diabetes } \\
\text { education class }\end{array}$ & Quality of Life \\
\hline $\begin{array}{l}\text { Thomas } \\
\text { Hong Kong } \\
(2012)\end{array}$ & Cluster & 72.1 & 66.2 & Healthy elderly & $\begin{array}{l}\text { Pedometry use plus } \\
\text { individual and group } \\
\text { support for physical } \\
\text { activity motivation } \\
\text { by 'peer buddy' } \\
\text { supporters }\end{array}$ & $\begin{array}{l}\text { 1) Non-pedometry } \\
\text { 2) Non-peer support } \\
\text { 3) Non-pedometry } \\
\text { and non-peer support }\end{array}$ & Physical activity \\
\hline $\begin{array}{l}\text { White }^{(44)} \\
\text { Australia } \\
(2012)\end{array}$ & Parallel & 64.6 & 40.5 & $\begin{array}{c}\text { Outpatients } \\
\text { with recent } \\
(<3 \text { months }) \\
\text { colorectal cancer } \\
\text { diagnosis }\end{array}$ & $\begin{array}{l}\text { Telephone support } \\
\text { to address (pre- } \\
\text { identified unmet } \\
\text { health needs) by } \\
\text { peer supporters }\end{array}$ & $\begin{array}{l}\text { Usual care (patients } \\
\text { informed of this } \\
\text { allocation) }\end{array}$ & $\begin{array}{l}\text { Proportion } \\
\text { Depressed } \\
\text { and Anxious } \\
\text { (not pooled) }\end{array}$ \\
\hline $\begin{array}{l}\text { Weber }^{(43)} \\
\text { USA } \\
(2007)\end{array}$ & Parallel & 60.0 & 0.0 & $\begin{array}{l}\text { Prostate cancer } \\
\text { patients } 6 \text { weeks } \\
\text { post radical } \\
\text { prostatectomy }\end{array}$ & $\begin{array}{l}\text { One-to-one } \\
\text { in-person discussions } \\
\text { (thoughts, feelings, } \\
\text { surgical side effects) } \\
\text { by peer supporters }\end{array}$ & $\begin{array}{l}\text { Usual care (provided } \\
\text { by urologist) }\end{array}$ & Depression \\
\hline
\end{tabular}

Volunteers were most commonly described as 'peers' in various roles including: general support, ${ }^{(34,38,43,45,48)}$ mentoring, ${ }^{(32,41,55,57)}$ educating, ${ }^{(37)}$ coaching, ${ }^{(39,41)}$ advising, ${ }^{(45)}$ and counseling. ${ }^{(40,42)}$ Volunteers were frequently described as non-proessionally trained, lay-tutors, ${ }^{(31)}$ lay health workers, ${ }^{(50)}$ and general 'volunteers', 55,57$)$ as well as variously described as: retired seniors, ${ }^{(35,52)}$ befrienders, ${ }^{(48,55,57)}$ volunteer professors, ${ }^{(53)}$ students, ${ }^{(49)}$ and facilitators ${ }^{(55,57)}$ (Table 1). Two of the included studies reported impact on senior volunteers themselves who described value and meaning in their own engagement with older adults, relating to their ability to connect and make a contribution. ${ }^{(48,57)}$

Study interventions intended to support a range of health goals, most commonly improving physical activity levels, ${ }^{35,41,42,50,51,54,56)}$ followed by chronic disease self-management skills, ${ }^{(31-33,37-39)}$ and coping with cancer, $(40,41,43,44)$ 
care giving, ${ }^{(48)}$ and end of life state, ${ }^{(52)}$ as well as improving overall mental well-being, ${ }^{(36,42,49)}$ social connectedness, ${ }^{(55,57)}$ and capacity for aging at home ${ }^{(55,57)}$ (Table 1$)$.

Blinding of participants is not considered feasible in this context and, therefore, the main concerns of bias were related to lack of assessor blinding affecting 22 included studies, ${ }^{(31,34-40,42-45,49-58)}$ inadequate allocation concealment in 11 studies, ${ }^{(31,36,37,40,42-45,49,52,53)}$ and incomplete outcome data ( $>20 \%$ of participants) in 9 studies ${ }^{(38,44,47,50,52,54-57)}$ (Figure 2) with individual study risk of bias reported in Appendix C.

\section{Physical Health}

Meta-analysis of 12 trials $(n=1,521)$ that reported physical functioning ${ }^{(31-36,41,47,49,51,54,57)}$ showed that participants who received volunteer support had statistically significant improvement in physical function compared to those who received usual care $(\mathrm{MD}=3.1$ points [95\% CI: 0.87 to 5.24 points] on a 100-point SF-36 physical component score; $\mathrm{I}^{2}=$ $72.0 \%$, low certainty evidence) (Figure 3, Table 2). We found no evidence of subgroup effect (Appendix D, Figures D.1, D.2, D.3, D.4) or a small study effect ( $p$ value for the Egger's test $=0.089$, Appendix D, Figure D.5).

Among six included RCTs $(n=1,349)$ that reported physical activity, meta-analysis showed that participants who received volunteer support had statistically significant improvement in their physical activity levels compared to those who received usual care or no physical activity intervention $(41,42,45,46,51,56)$ $\left(\mathrm{SMD}=0.5\right.$ points $[95 \% \mathrm{CI}: 0.14$ to 0.83$] ; \mathrm{I}^{2}=83.7 \%$, low certainty evidence) (Figure 4, Table 2). We found no evidence of subgroup effect for adequate allocation concealment or blinding of assessors (Appendix E, Figures E.1, E.2).

\section{Mental Health}

Meta-analysis of 10 trials $(\mathrm{n}=1,341)$ that reported emotional functioning ${ }^{(31-36,41,47,51,54,57)}$ showed no difference between participants receiving volunteer support compared to those who received usual care $(\mathrm{MD}=-0.34$ points [95\% CI: -1.22 to 0.54 points] on a 100 -point SF-36 mental component score; $\mathrm{I}^{2}=0 \%$, low certainty evidence) (Figure 5 , Table 2 ). We found no evidence of subgroup effect (Appendix F, Figures F.1, F.2, F.3, F.4) or small study effect ( $p$ value for the Egger's test = 0.593, Appendix F, Figure F.5).

Meta-analysis of 11 trials $(n=1,382)$ that reported depression scores ${ }^{(31,32,34-36,43,47,48,52,53,57)}$ showed no difference between participants receiving volunteer support compared to those who received usual care $(\mathrm{MD}=0.3$ points lower $[95 \%$ CI: -1.17 to 0.58 points] on a 10-point HADS-depression subscale; $\mathrm{I}^{2}=0 \%$, low certainty evidence) (Figure 6, Table 2 ). We found no evidence of subgroup effect (Appendix G, Figures G.1, G.2, G.3, G.4) or small study effect ( $p$ value for the Egger's test $=.356$, Appendix G, Figure G.5).

Meta-analysis of 5 trials $(n=920)$ that reported anxiety scores $^{(31,32,40,47,48)}$ showed no difference between participants receiving volunteer support compared to those who received usual care ( $\mathrm{MD}=0.04$ points lower [ $95 \% \mathrm{CI}$ : $-0,56$ to 0.65 points] on the 10-point HADS-anxiety subscale; $\mathrm{I}^{2}=33.8 \%$, low certainty evidence) (Figure 7, Table 2). We found no evidence of subgroup effect (Appendix H, Figures H.1, H.2, H.3).

\section{Quality of Life}

Meta-analysis of 8 studies $(n=1,437)$ that reported quality of life ${ }^{(34,35,38,39,48,51,54,57)}$ showed no difference between participants receiving volunteer support compared to those who received usual care or no intervention ( $\mathrm{MD}=0.00$ points

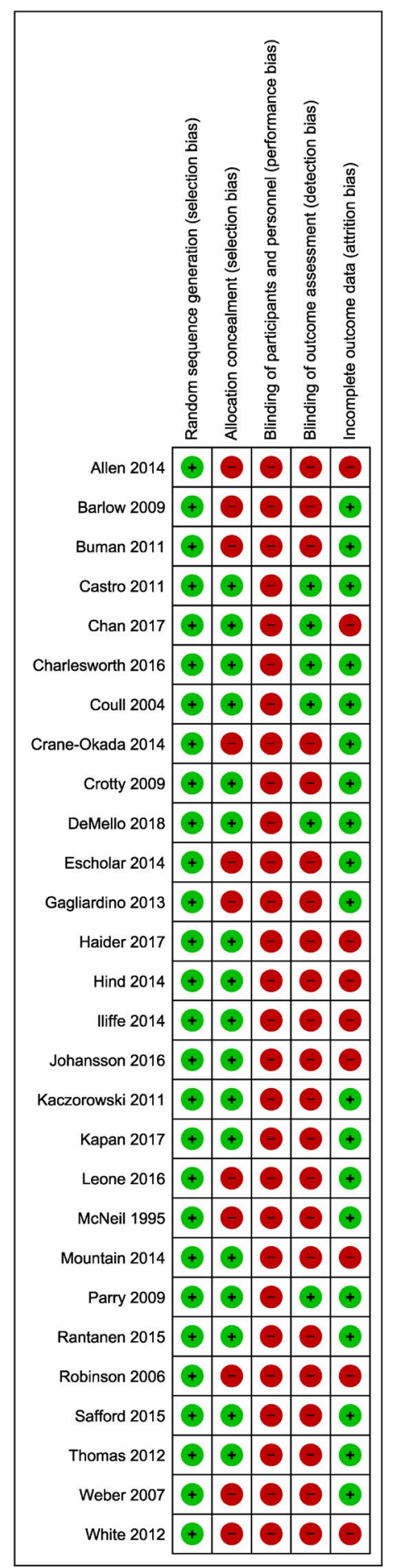

FIGURE 2. Risk of bias-included studies 
MOORE: VOLUNTEER IMPACT ON SENIORS

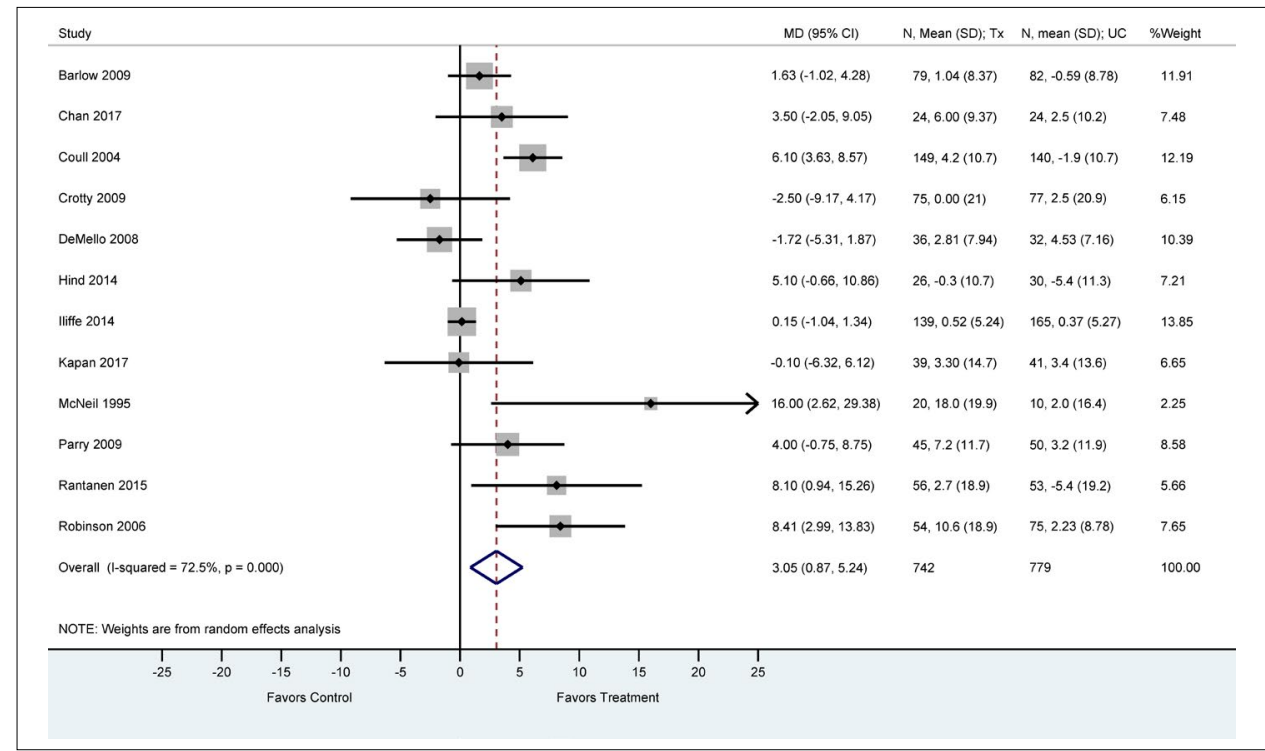

FIGURE 3. Physical function (mean difference SF-36 PCS)

TABLE 2.

GRADE summary of findings

\begin{tabular}{|c|c|c|c|}
\hline $\begin{array}{l}\text { Outcome } \\
\text { Timeframe }\end{array}$ & Study Results and Measurements & $\begin{array}{c}\text { Absolute Effect Estimates } \\
\text { Usual Care Volunteers }\end{array}$ & Certainty of Evidence \\
\hline \multirow{3}{*}{$\begin{array}{c}\text { Anxiety }^{\mathrm{a}} \\
\text { Longest follow-up }\end{array}$} & \multirow{3}{*}{$\begin{array}{c}\text { Measured by: HADS-A } \\
\text { Scale: } 11-21 \text { Lower better } \\
\text { Based on data from } 920 \text { patients in } 5 \text { studies } \\
\text { Follow up longest follow-up } \\
\text { (average } 34.4 \text { wks) }\end{array}$} & 0.32 & \multirow{3}{*}{$\begin{array}{c}\text { Moderate } \\
\text { Due to serious risk } \\
\text { of bias }^{\mathrm{b}}\end{array}$} \\
\hline & & Mean & \\
\hline & & $\begin{array}{l}\text { Difference: MD } 0.04 \text { lower } \\
\text { (CI 95\% 0.56 higher to } 0.65 \text { lower) }\end{array}$ & \\
\hline \multirow{3}{*}{$\begin{array}{c}\text { Depression }^{\mathrm{c}} \\
\text { Longest follow-up }\end{array}$} & \multirow{3}{*}{$\begin{array}{c}\text { Measured by: HADS-D } \\
\text { Scale: 11-21 Lower better } \\
\text { Based on data from } 1382 \text { patients } \\
\text { in } 11 \text { studies } \\
\text { Follow up longest follow-up } \\
\text { (average } 24.2 \text { wks) }\end{array}$} & 0.16 & \multirow{3}{*}{$\begin{array}{l}\text { Low } \\
\text { Due to serious risk of } \\
\text { bias, Due to serious } \\
\text { imprecision }^{\mathrm{d}}\end{array}$} \\
\hline & & Mean & \\
\hline & & $\begin{array}{l}\text { Difference: MD } 0.27 \text { lower } \\
\text { (CI } 95 \% 0.03 \text { higher to } 0.57 \text { lower) }\end{array}$ & \\
\hline \multirow{3}{*}{$\begin{array}{c}\text { Emotional } \\
\text { Functioning } \\
\text { Longest follow-up }\end{array}$} & \multirow{3}{*}{$\begin{array}{l}\text { Measured by: Mental Component } \\
\text { Summary score (SF-36) } \\
\text { Scale: } 0-100 \text { High better } \\
\text { Based on data from } 1341 \text { patients } \\
\text { in } 10 \text { studies } \\
\text { Follow up longest follow-up } \\
\text { (average } 26.6 \text { wks) }\end{array}$} & 1.84 & \multirow{3}{*}{$\begin{array}{l}\text { Moderate } \\
\text { Due to serious risk } \\
\text { of bias }^{f}\end{array}$} \\
\hline & & Mean & \\
\hline & & $\begin{array}{l}\text { Difference: MD -0.34 lower } \\
\text { (CI 95\% } 1.22 \text { lower to } 0.54 \text { higher) }\end{array}$ & \\
\hline \multirow{4}{*}{$\begin{array}{c}\text { Physical } \\
\text { Functioning } \\
\text { Longest follow-up }\end{array}$} & \multirow{4}{*}{$\begin{array}{l}\text { Measured by: Physical Component } \\
\text { Summary score (SF-36) } \\
\text { Scale: 0-100 High better } \\
\text { Based on data from } 1521 \text { patients } \\
\text { in } 12 \text { studies } \\
\text { Follow up longest follow-up } \\
\text { (average } 25.1 \mathrm{wks} \text { ) }\end{array}$} & 0.62 & \multirow{4}{*}{$\begin{array}{l}\text { Low } \\
\text { Due to serious risk of } \\
\text { bias, Due to serious } \\
\text { inconsistency leading } \\
\text { to imprecision }\end{array}$} \\
\hline & & Mean & \\
\hline & & \multirow[t]{2}{*}{$\begin{array}{l}\text { Difference: MD } 3.05 \text { higher } \\
\text { (CI } 95 \% 0.87 \text { higher to } 5.24 \text { higher) }\end{array}$} & \\
\hline & & & \\
\hline \multirow{3}{*}{$\begin{array}{l}\text { Quality of life }{ }^{\mathrm{i}} \\
\text { Longest follow-up }\end{array}$} & \multirow{3}{*}{$\begin{array}{c}\text { Measured by: EQ-5D total score } \\
\text { Scale: 0-1 High better } \\
\text { Based on data from } 1437 \text { patients in } 8 \text { studies } \\
\text { Follow up longest follow-up } \\
\text { (average } 39.2 \mathrm{wks} \text { ) }\end{array}$} & -0.02 & \multirow{3}{*}{$\begin{array}{l}\text { Low } \\
\text { Due to serious risk of } \\
\text { bias, and publication } \\
\text { bias (i.e. small } \\
\text { study effect) }\end{array}$} \\
\hline & & Mean & \\
\hline & & $\begin{array}{l}\text { Difference: MD } 0.00 \text { lower } \\
\text { (CI 95\% } 0.02 \text { lower to } 0.01 \text { higher) }\end{array}$ & \\
\hline
\end{tabular}


MOORE: VOLUNTEER IMPACT ON SENIORS

TABLE 2. Continued

\begin{tabular}{|c|c|c|c|}
\hline $\begin{array}{l}\text { Outcome } \\
\text { Timeframe }\end{array}$ & Study Results and Measurements & $\begin{array}{c}\text { Absolute Effect Estimates } \\
\text { Usual Care Volunteers }\end{array}$ & Certainty of Evidence \\
\hline $\begin{array}{l}\text { Physical Activity } \\
\text { Longest follow-up }\end{array}$ & $\begin{array}{l}\text { Measured by: MET (energy } / \mathrm{kg} / \mathrm{mns} / \mathrm{wk} \text { ); } \\
\text { MVPA per week; minutes spent on exercise } \\
\text { Scale: - High better } \\
\text { Based on data from } 1349 \text { patients in } \\
6 \text { studies (average } 10.2 \text { months) }\end{array}$ & $\begin{array}{c}\text { Mean } \quad \text { Mean } \\
\text { Difference: SMD 0.48 more } \\
\text { (CI 95\% 0.14 more - } 0.83 \text { more) }\end{array}$ & $\begin{array}{l}\text { Low } \\
\text { Due to serious risk of } \\
\text { bias and indirectness }^{\mathrm{k}}\end{array}$ \\
\hline $\begin{array}{l}\text { Frequency } \\
\text { of Hospital } \\
\text { Admissions }\end{array}$ & $\begin{array}{l}\text { Measured by: Narrative report: } \\
\text { Admission rate not provided }{ }^{(37)} \\
\text { and mean hospital admission rate } \\
\text { per } 1000 \text { participants }{ }^{(58)}\end{array}$ & $\begin{array}{l}2 \text { studies reported hospitalization } \\
\text { frequency. One qualitative report of } \\
\text { no significant difference between } \\
\text { groups. }{ }^{(37)} \text { Another study reported } \\
\text { the incidence of hospitalization as } \\
(27.9 / 1000) \text { in the intervention group } \\
\text { versus }(30.13 / 1000) \text { control group } \\
(p=<.01)\end{array}$ & $\begin{array}{l}\text { Low } \\
\text { Due to serious risk of } \\
\text { bias and inconsistency }{ }^{1}\end{array}$ \\
\hline Falls & $\begin{array}{l}\text { Measured by: Narrative report: } \\
\text { Proportion of participants reporting one } \\
\text { or more falls in the past } 3 \text { months (fallers) } \\
\text { and the incidence of falls }\end{array}$ & $\begin{array}{l}2 \text { studies reported falls. One RCT } \\
\text { reported the difference between } \\
\text { proportion of fallers in the intervention } \\
\text { group }(14 / 35) \text { versus }(8 / 19) \text { in the } \\
\text { control group }(\mathrm{P}=0.11)^{(51)} \text { Another } \\
\text { study (cluster } \mathrm{RCT}) \text { reported the } \\
\text { incidence of falls in the intervention } \\
\text { population }(100 / 183) \text { versus }(158 / 217) \\
\text { in the control population }(\mathrm{p}=<.01)^{(54)}\end{array}$ & $\begin{array}{l}\text { Low } \\
\text { Due to serious } \\
\text { risk of bias and } \\
\text { inconsistency }^{\mathrm{m}}\end{array}$ \\
\hline Adverse Events & Narrative summary (results not pooled) & $\begin{array}{l}6 \text { studies reported adverse events, no } \\
\text { events or no difference between groups } \\
\text { was found }{ }^{(32,34,46,54,57)}\end{array}$ & $\begin{array}{l}\text { Low } \\
\text { Due to serious risk of } \\
\text { bias and inconsistency }{ }^{\mathrm{n}}\end{array}$ \\
\hline
\end{tabular}

HADS = Hospital Anxiety-Depression-Depression; HADS-A = Hospital Anxiety Depression-Anxiety; MET = Metabolic Equivalent Task, Energy used/per $\mathrm{Kg} /$ minute/week; MVPA + Time spent in moderate to vigorous physical activity.

${ }^{\mathrm{a} A l l}$ Measures converted to HADS-A.

${ }^{b}$ Anxiety: Risk of bias: Serious. Inadequate/lack of blinding of outcome assessors, resulting in potential for detection bias, Incomplete data and/or large loss to follow up; Inconsistency: Serious. Imprecision: Not serious. Wide confidence intervals; decided not to rate down further for imprecision as it is due to inconsistency.; Publication bias: Not serious. Not assessed due to small number of studies.

${ }^{\mathrm{c} A l l}$ measures converted to HADS-D.

${ }^{\mathrm{d}}$ Depression: Risk of bias: Serious. Inadequate/lack of blinding of outcome assessors, resulting in potential for detection bias, Incomplete data and/or large loss to follow up.; Inconsistency: Serious. Point estimates vary widely, The confidence interval of some of the studies do not overlap with those of most included studies/ the point estimate of some of the included studies.; Imprecision: Not serious. Decided not to rate down for imprecision as it is mostly due to inconsistency.

${ }^{e}$ Emotional Function: Risk of bias: Serious. Inadequate concealment of allocation during randomization process, resulting in potential for selection bias, Inadequate/lack of blinding of outcome assessors, resulting in potential for detection bias, Incomplete data and/or large loss to follow up; Inconsistency: Not serious. Decided not to rate further down as the observed heterogeneity seems to be due to risk of bias; Imprecision: Serious. Wide confidence intervals. ${ }_{\mathrm{f}}^{\mathrm{A}}$ All measures converted to PCS score.

gPhysical Function: Risk of bias: Serious. Inadequate concealment of allocation during randomization process, resulting in potential for selection bias, Inadequate/lack of blinding of outcome assessors, resulting in potential for detection bias, Incomplete data and/or large loss to follow up.

${ }^{\mathrm{h}}$ All measures converted to EQ-5D total score.

i Quality of Life: Risk of bias: Serious. Incomplete data and/or large loss to follow up; significant test of interaction for the subgroup of low vs. high risk of bias due to missing participants data.; Publication bias: Serious. Asymmetrical funnel plot with evidence of small study effect.

jPhysical Activity: Risk of bias: Serious. Inadequate concealment of allocation during randomization process, resulting in potential for selection bias, Inadequate/lack of blinding of outcome assessors, resulting in potential for detection bias, Incomplete data and/or large loss to follow up; Inconsistency: Not serious. Decided not to rate further down as the observed heterogeneity seems to be due to risk of bias; Indirectness: Serious, Publication bias: Not serious. Less than 10 studies.

${ }^{k}$ All measures converted to MCS.

${ }^{1}$ Hospital admission: Risk of bias: Serious. Inadequate concealment of allocation during randomization process, resulting in potential for selection bias, inadequate/lack of blinding of outcome assessors; Inconsistency: Serious Uncertain effects narrative summary.

mFalls: Risk of bias: Serious. Inadequate concealment of allocation during randomization process resulting in potential for selection bias; Inconsistency: Serious. Uncertain effects with narrative summary.

${ }^{\mathrm{n}}$ Averse events: Risk of bias: Serious. Inadequate/lack of blinding of assessors resulting in potential for detection bias, incomplete outcome reporting. Inconsistency: Serious Uncertain effects narrative summary. 
[95\% CI: -0.02 to 0.01 points] on a 1 point EQ-5D Scale; $\mathrm{I}^{2}$ $=0 \%$, low certainty evidence) (Figure 8 , Table 2). We found evidence of a significant test of interaction for the subgroup of low versus high risk of bias due to missing participants data $(p=.012)$. Studies at low risk of bias for missing participant data were not significantly associated with volunteer improvement in Quality of Life $(>20 \%)(\mathrm{MD}=0.02$ points $[95 \% \mathrm{CI}$ : 0.00 to 0.05 points] $) ; \mathrm{I}^{2}=0 \%, p=.917$. No other evidence for subgroup effects were noted (Appendix I, Figures I.1, I.2, I.3). Small study effect (publication bias) was not detected $($ Eggers Test $=0.062)($ Appendix I, Figure I.4)

\section{Falls}

Two included RCTs reported falls using different metrics, the incidence of falls ${ }^{(54)}$ and the proportion of fallers, ${ }^{(51)}$ and were not pooled, but summarized narratively, as follows. For frail older adults, a 12-week structured physical training and nutrition intervention carried out by lay volunteers showed a decrease in the proportion of fallers, but not reaching significance $(p=.11){ }^{(51)}$ For seniors over 65 years of age drawn from a general practice population (stable chronic health conditions), a class-based community falls management exercise program delivered by peer mentors significantly reduced

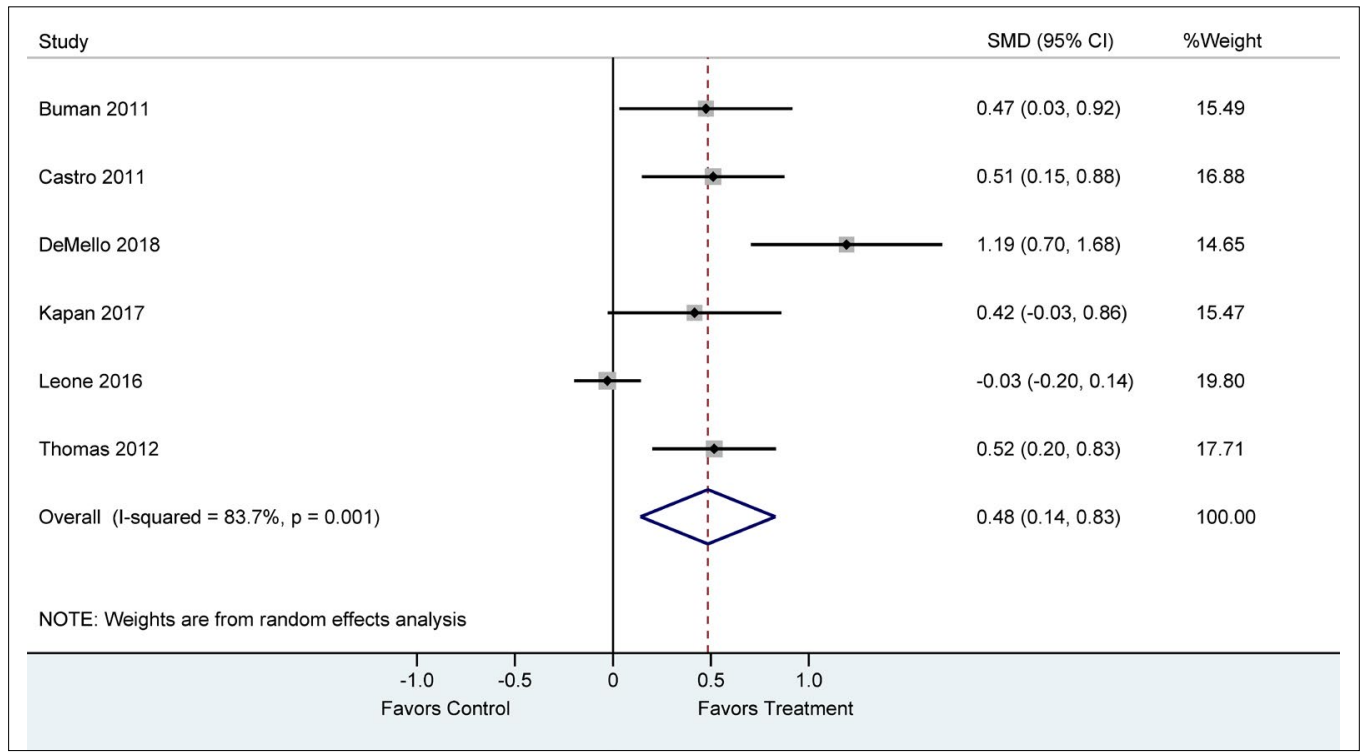

FIGURE 4. Physical activity (standardized mean differences)

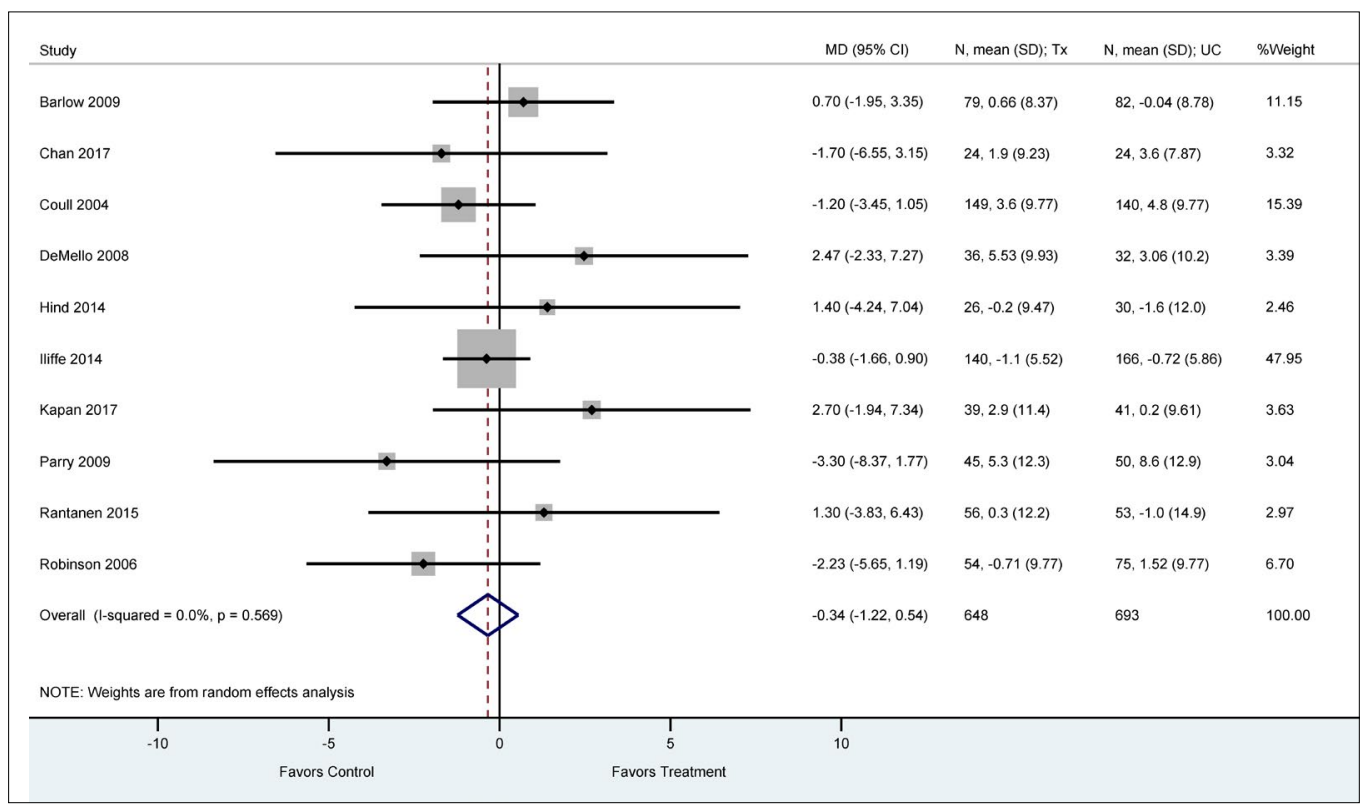

FIGURE 5. Emotional function (mean difference SF-36 MCS) 
falls by $18 \%(p<.01)^{(54)}$ and increased self-reported physical activity 12 months after the intervention.

\section{Hospitalization}

Two included RCTs reporting hospitalization were not pooled, as one study reported the mean admission rate per 1000 population $^{(58)}$ and the other provided a descriptive report of admission (quantitative rate not provided). ${ }^{(37)}$ A cluster randomized trial of a cardiovascular health awareness program delivered by volunteers versus no intervention reported a $9 \%$ adjusted relative reduction in cardiac-related admissions (95\% confidence interval 0.86 to $0.97 ; p=.002$ ), although all cause admissions were not reduced. ${ }^{(58)}$ A diabetes education program provided by professionals versus peer volunteers reported that "few hospitalizations were recorded in the overall population sample, with no significant difference between groups during the study."(37)

\section{Adverse Events}

Six included studies reported surveillance for adverse events or harms, three reported no adverse events occurring for patients or volunteers, ${ }^{(34,46,57)}$ and two studies reported no difference between study arms. ${ }^{(32,54)}$ One non-critical event occurred when one volunteer experienced discomfort when a caregiver stepped out of the boundaries of a befriending role. ${ }^{(48)}$

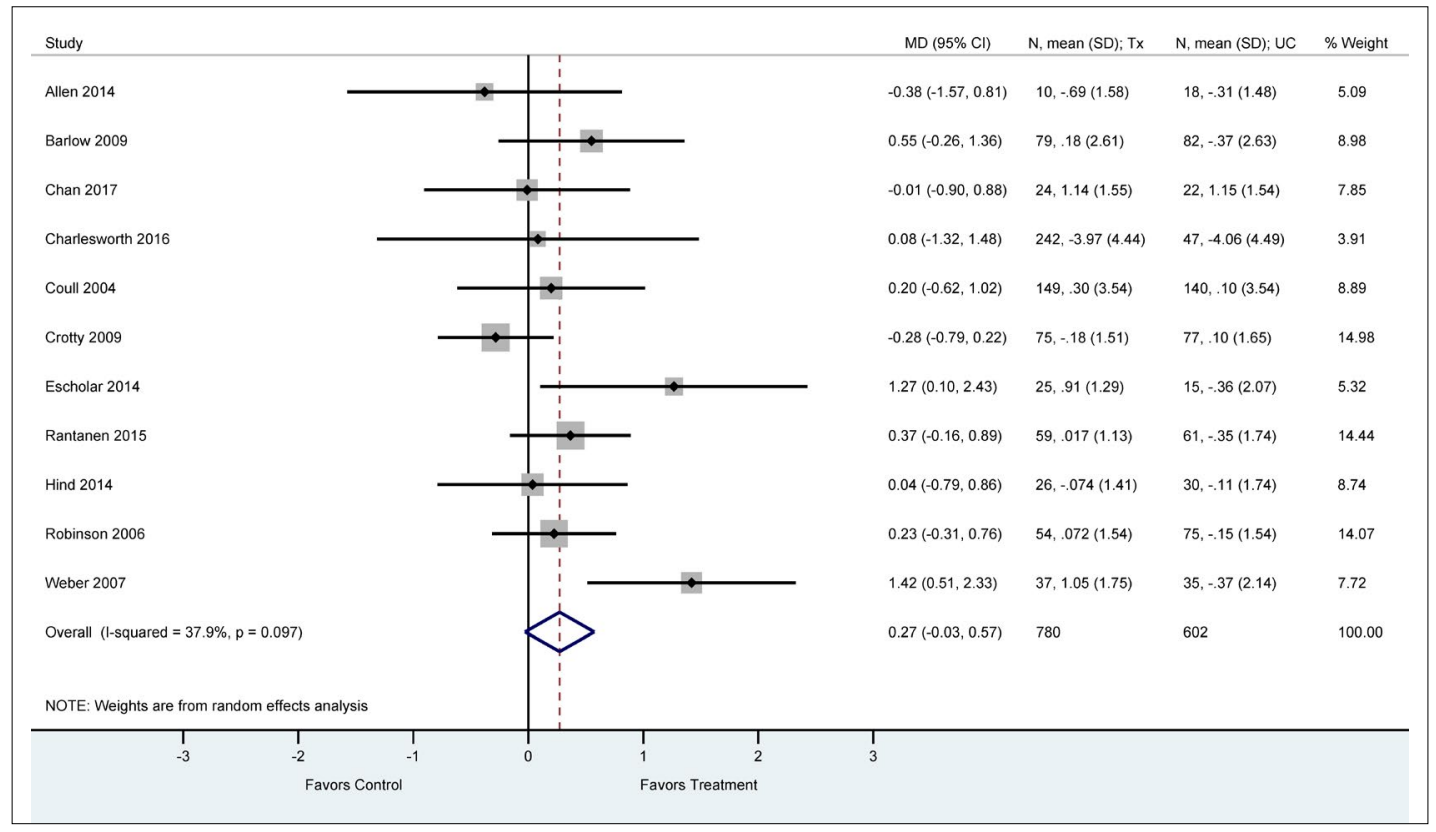

FIGURE 6. Depression (mean difference HADS)

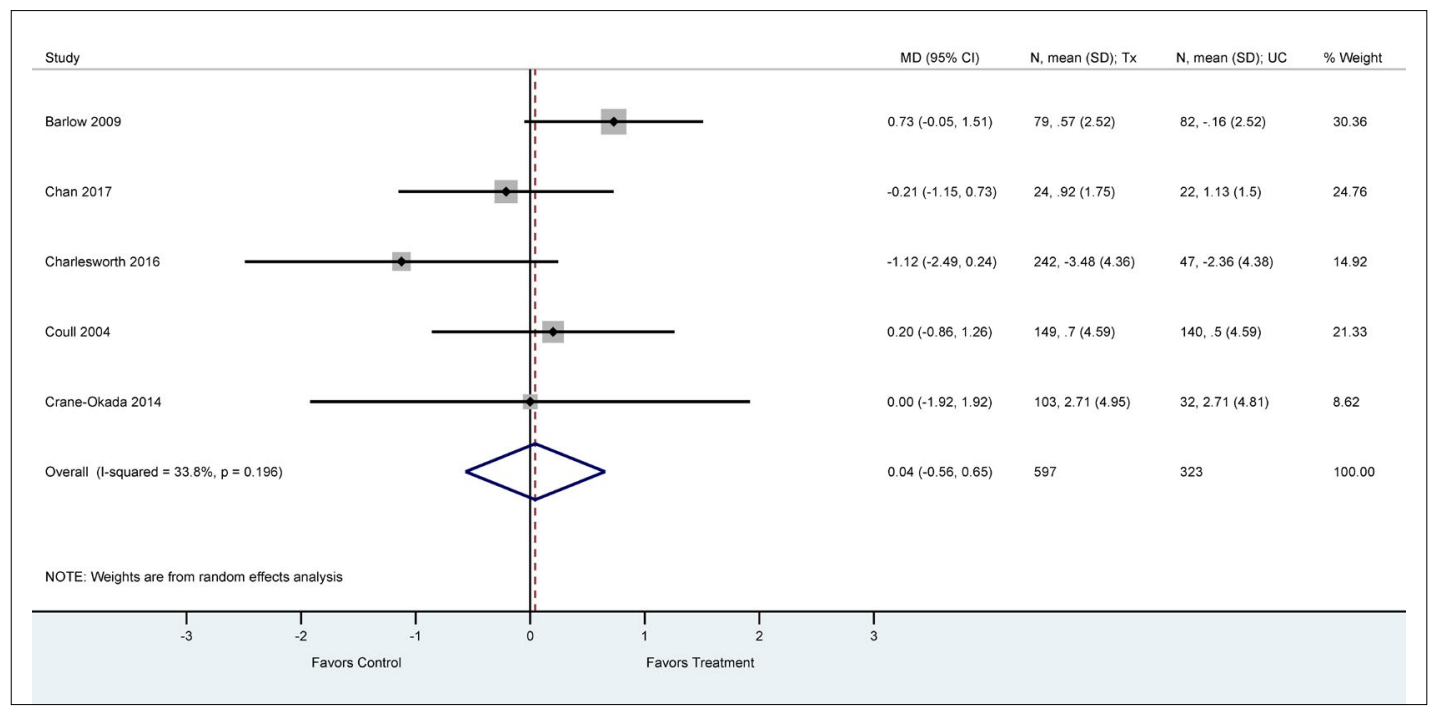

FIGURE 7. Anxiety (mean difference HADS) 
MOORE: VOLUNTEER IMPACT ON SENIORS

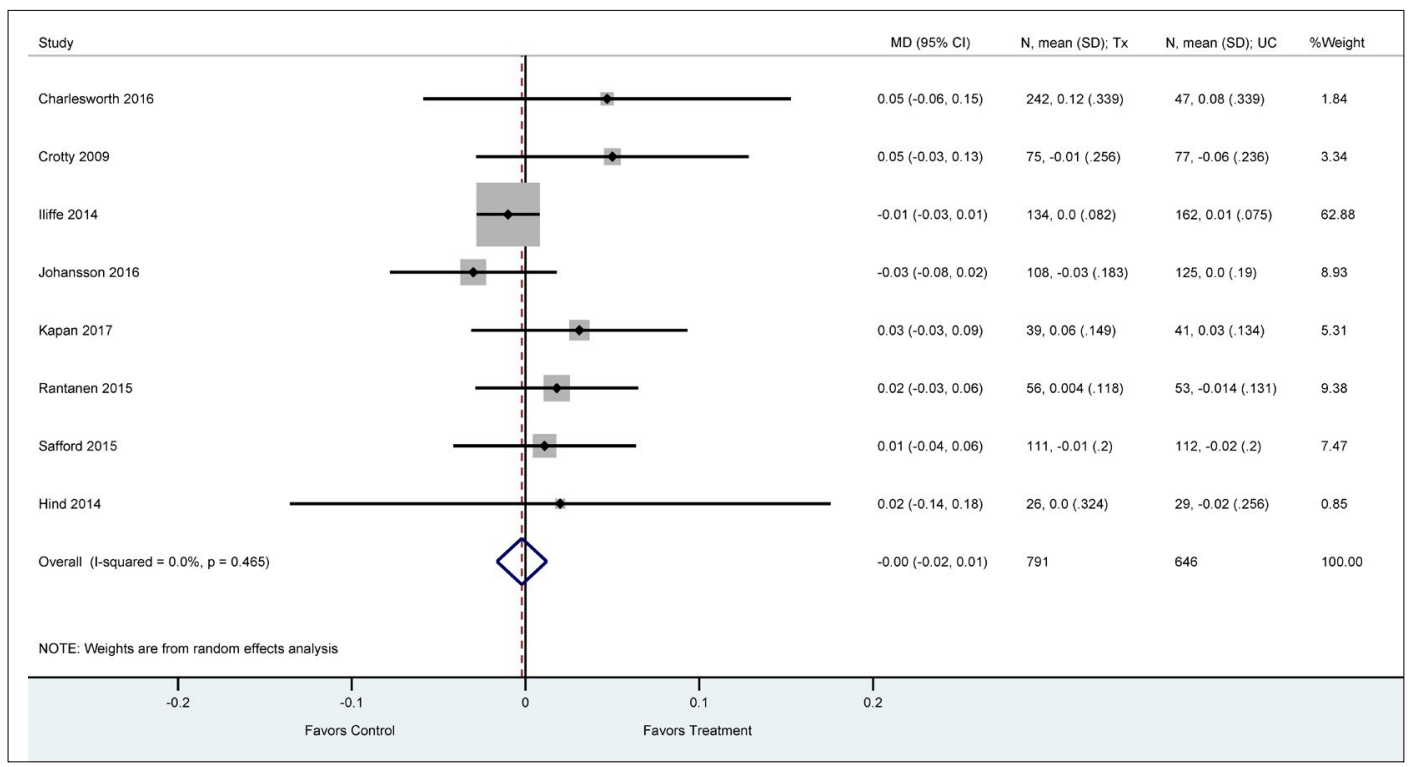

FIGURE 8. Quality of life (mean difference EQ5D)

\section{DISCUSSION}

We identified 27 unique RCTs addressing the impact of unpaid volunteers on the health of older adults residing in the community, almost all taking place in high-income countries, focusing on seniors living with a range of health conditions and health states. Volunteers addressed a diversity of health needs and goals and represented a variety of roles. Despite this diversity (moderate to high heterogeneity) that was not explained by study risk of bias or participants' age or sex, our results support the role of volunteers to improve physical function and physical activity levels for seniors. This benefit for increased physical activity and self-reported physical health was identified regardless of the health condition being targeted (e.g. recent myocardial infarction, ${ }^{(31,32)}$, dysphoria, ${ }^{(49)}$ severe physical disabilities, ${ }^{(36)}$ cancer, ${ }^{(40-42,44)}$ or recent hip surgery, ${ }^{(34)}$ or the volunteer role provided (e.g., lay tutor, ${ }^{(31)}$ lay mentor, ${ }^{(32)}$ volunteer student, ${ }^{(49)}$ or peer support ${ }^{(36,38)}$ ).

Analysis for depression trended toward favouring volunteer support, although, it did not reach statistical significance. There was no volunteer effect noted for anxiety or for senior's emotional function. Anxiety levels were particularly worse for carers of people with dementia who were supported by friendship volunteers (compared to usual care), and this study appears to be driving the overall effect on anxiety; however, anxiety was a secondary outcome in this study. Of note, depression scores from this study (a primary outcome) approached statistically significant improvement for these befriended carers $(95 \% \mathrm{CI}-0.09$ to $2.84, p=.06)$. (48) Quality of life as measured by five studies with low risk of bias for missing participant data $(<20 \%)$ trended toward a volunteer effect, although it was not significant. For the two studies which reported falls, the incidence of falls was significantly reduced in one, but the proportion of fallers was not significantly reduced in the other. ${ }^{(51,54)}$ This may be explained by differences in the study population and/or differences in the type and duration of volunteer intervention. The proportion of fallers among frail elderly individuals who received home-based physical training from volunteer buddies for 12 weeks was not significantly reduced. ${ }^{(51)}$ Whereas the number of falls was significantly reduced for more robust seniors who received a class-based community exercise plus walking program for 24 weeks. ${ }^{(54)}$ Hospitalization rates were no different for professional diabetic educators compared to peer volunteer educators, ${ }^{(37)}$ while cardiovascular related admissions were significantly reduced for a volunteer delivered, communitybased cardiovascular awareness intervention. ${ }^{(58)}$ Adverse events were monitored in six studies and reported as either no events or no significant event difference. Heterogeneity was not explained by any analyses conducted.

\section{Findings in Context}

To our knowledge this is the first review to specifically synthesize trial-level data for the impact of unpaid volunteers on health-related outcomes for older adults living in the community with a variety of primary care conditions. Although indirect from our intervention of interest for unpaid volunteers, one review that focused on peer-supporters (paid and unpaid) for those living with diabetes (no age specification), also found a positive association with improvements in physical activity. ${ }^{(59)}$ Other reviews of paid lay health workers in primary and community care provided a narrative report of improved health-related behavior, including increased physical activity, ${ }^{(60)}$ with mixed results for mental and physical function. (13) Other outcomes of interest were not summarized in these reviews, however participants were generally satisfied with lay health worker encounters and increased their knowledge of disease and self-management. ${ }^{(60)}$ 
These findings of physical health benefit have implications for functional ability and independence for older adults in the community. Both regular physical activity and short- term exercise programs are associated with significantly reduced risk of functional limitations and disability in older adults across a range of functional measures. ${ }^{(61)}$ Relatedly, robust evidence from two Cochrane reviews support exercise as effective falls prevention interventions; ${ }^{(62,63)}$ this was achieved with only half of community-dwelling older participants adhering to exercise interventions. ${ }^{(61)}$ Since falls represent the leading cause of fatal and non-fatal injuries among adults aged 65 and older, it is conceivable that trained volunteers supporting adherence to exercise guidance ${ }^{(62)}$ could reduce falls and associated disability, thereby maintaining independence for aging in place. Consistent with this supposition, we also found that falls were significantly reduced for communitybased seniors over 65 years of age (with stable chronic health conditions), who received a six-month falls management exercise program, ${ }^{(54)}$ and for frail elderly receiving a 12 -week structured physical training and nutrition program, although not reaching significance in this population $(p=.10) .{ }^{(51)}$

Although heterogeneity across volunteer interventions limits identification of specific predictors of improved health outcomes, the observed benefit may be attributed to both the natural motivation of volunteers to help, and to the frequently used volunteer interventions of informational and emotional support, social connection and feedback on goal progress, which are consistent with social support ${ }^{(64)}$ and self-efficacy theories. ${ }^{(65)}$ Given that analyses for depression and possibly quality of life (considering low risk of bias studies) favoured volunteer interventions but were not statistically significant, further study of how volunteers can be best integrated into delivery processes of community-based care is warranted.

\section{Limitations}

Certainty of evidence was low mainly due to high risk of bias and inconsistency, and generalizability is limited to highincome countries. Heterogeneity (moderate to high) was not explained by study risk of bias items, imputed variability estimates, mean participant age, or proportion of female participants. Diverse volunteer characteristics and contexts (e.g., roles, activities, volunteer support and training, recipient health conditions, underlying theoretical basis for volunteer interventions), as well as the variety of terms used to describe such volunteer characteristics, limited subgroup analyses of 'like' studies that would allow for inference about volunteer variables and their impact on outcomes of interest. Agreed upon terms and definitions to describe volunteers (e.g., peer, mentor, counselor, tutor, educator, buddy, befriender, facilitator, guide), as well as development of a volunteer taxonomy (e.g., roles, activities, theoretical basis for volunteer interventions, duration of volunteer training and contact with recipients), would allow for better understanding of optimal volunteer conditions and their impacts.

\section{CONCLUSIONS}

We found evidence to support the role of volunteers to increase physical activity levels for seniors and to improve their subjective ratings of physical health, without harm. As relevant indicators of therapeutic success, particularly for independent living in older people, these findings align with the WHO call to action on aging. Policymakers, clinical leaders, health system planners, volunteer organizations, and others could make use of this synthesized evidence to consider the role of volunteers in health system planning for aging populations.

\section{ACKNOWLEDGEMENTS}

We wish to acknowledge the following individuals for their assistance with database searching and initial study screening: Mehreen Bhamani, Jennifer Longaphy, Steve Dragos, Stephanie Di Pelino, and Fiona Parascandalo. We are particularly grateful to Lynda Nash for her logistics expertise and administrative leadership.

\section{CONFLICT OF INTEREST DISCLOSURES}

The authors declare that no conflicts of interest exist. 
MOORE: VOLUNTEER IMPACT ON SENIORS

APPENDIX A. Search strategy, including Ovid MEDLINE(R) and Epub Ahead of Print, In-Process \& Other Non-Indexed Citations, Daily and Versions(R), 1946 to November 1, 2018

\begin{tabular}{|c|c|c|}
\hline Searches & Results & Type \\
\hline 1 & Aged/ & 2832463 \\
\hline 2 & "Aged, 80 and over"/ & 819963 \\
\hline 3 & *Aging/ & 137261 \\
\hline 4 & *Geriatrics/ & 25515 \\
\hline 5 & ((55 year? or 65 year? or 75 year?) adj2 (above or older or over or plus)).ti,ab,kw. & 27254 \\
\hline 6 & ("55 and over" or "65 and over" or "75 and over").ti,ab,kw. & 3586 \\
\hline 7 & $\begin{array}{l}((\text { aged or elderly or geriatric* or old or older or senior?) adj2 (adult? or citizen? or individual? or } \\
\text { people or person?)).ti,ab,kw. }\end{array}$ & 193136 \\
\hline 8 & 1 or 2 or 3 or 4 or 5 or 6 or 7 & 3033016 \\
\hline 9 & * Community Health Workers / & 3151 \\
\hline 10 & *Health Auxiliary/ & 0 \\
\hline 11 & Hospital Volunteers/ & 1295 \\
\hline 12 & *Mentors/ & 5337 \\
\hline 13 & *Mentor/ & 5337 \\
\hline 14 & PEER GROUP/ & 18404 \\
\hline 15 & Counseling/ or Peer Group/ & 51411 \\
\hline 16 & Peers/ & 0 \\
\hline 17 & exp Peer Group/ & 18670 \\
\hline 18 & VOLUNTEERS/ & 9083 \\
\hline 19 & “Voluntary Worker”.mp. or Volunteers/ & 9087 \\
\hline 20 & (lay worker? or voluntary worker? or volunteer* or peer* or (train* adj2 student?)).ti,ab,kw. & 259231 \\
\hline 21 & 9 or 10 or 11 or 12 or 13 or 14 or 15 or 16 or 17 or 18 or 19 or 20 & 309427 \\
\hline 22 & 8 and 21 & 43546 \\
\hline 23 & limit 22 to humans & 42659 \\
\hline 24 & HOSPITALIZATION/ & 95288 \\
\hline 25 & Accidental Falls/ & 21340 \\
\hline 26 & "Quality of Life"/ & 168131 \\
\hline 27 & Mental Health/ & 32075 \\
\hline 28 & $\begin{array}{l}\text { Physical Health.mp. [mp=title, abstract, original title, name of substance word, subject heading } \\
\text { word, floating sub-heading word, keyword heading word, protocol supplementary concept word, rare } \\
\text { disease supplementary concept word, unique identifier, synonyms] }\end{array}$ & 17414 \\
\hline 29 & “EQ5D”.mp. & 456 \\
\hline 30 & Quality-Adjusted Life Years/ or Cost-Benefit Analysis/ or "Quality of Life"/ & 239968 \\
\hline 31 & 23 and $(24$ or 25 or 26 or 27 or 28 or 29 or 30$)$ & 2412 \\
\hline 32 & limit 31 to (humans and randomized controlled trial) & 565 \\
\hline 33 & limit 31 to (humans and systematic reviews) & 152 \\
\hline 34 & Exercise/ & 94722 \\
\hline 35 & Physical activity.mp. & 92922 \\
\hline 36 & 23 and $(24$ or 25 or 26 or 27 or 28 or 29 or 30 or 34 or 35$)$ & 3779 \\
\hline 37 & limit 36 to (humans and randomized controlled trial) & 879 \\
\hline 38 & limit 37 to (humans and systematic reviews) & 18 \\
\hline
\end{tabular}


APPENDIX B: Data extraction form

\begin{tabular}{|c|}
\hline Data Item \\
\hline Study ID \\
\hline $\begin{array}{c}\text { Study } \\
\text { (First Author Name) }\end{array}$ \\
\hline Trial Arm (Intervention/Control) \\
\hline Number Randomized \\
\hline Comments \\
\hline Scale (add outcome definition if necessary) \\
\hline $\begin{array}{c}\text { Direction of Scoring } \\
(1 \text { higher }=\text { better, } 2 \text { higher }=\text { worse })\end{array}$ \\
\hline Range of Scale \\
\hline Follow up Time (weeks) \\
\hline Other Follow Up Times \\
\hline Number Analyzed \\
\hline Baseline Mean \\
\hline Baseline SD \\
\hline Follow Up (Effect Size) \\
\hline Follow Up (Standard deviation) \\
\hline Change (Effect Size) \\
\hline Change (Standard Deviation) \\
\hline
\end{tabular}

APPENDIX C. Risk of bias (individual studies)

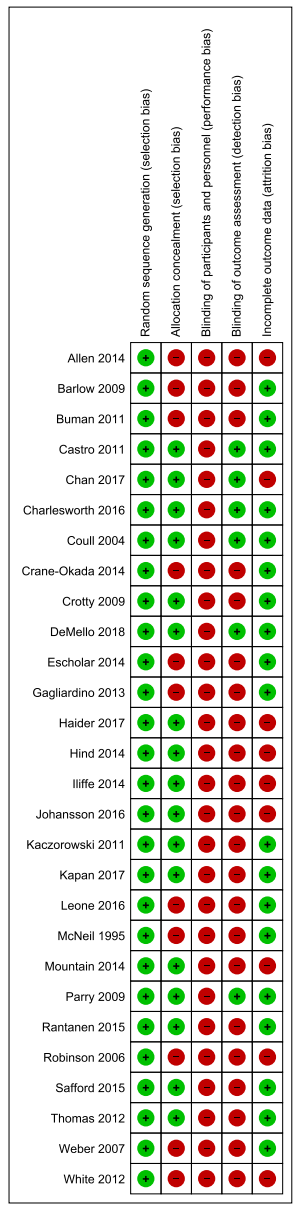

APPENDIX D. Physical function (mean difference SF-36 physical component score-100 point scale)

D.1 Physical function (subgroup analysis - allocation concealed adequately)

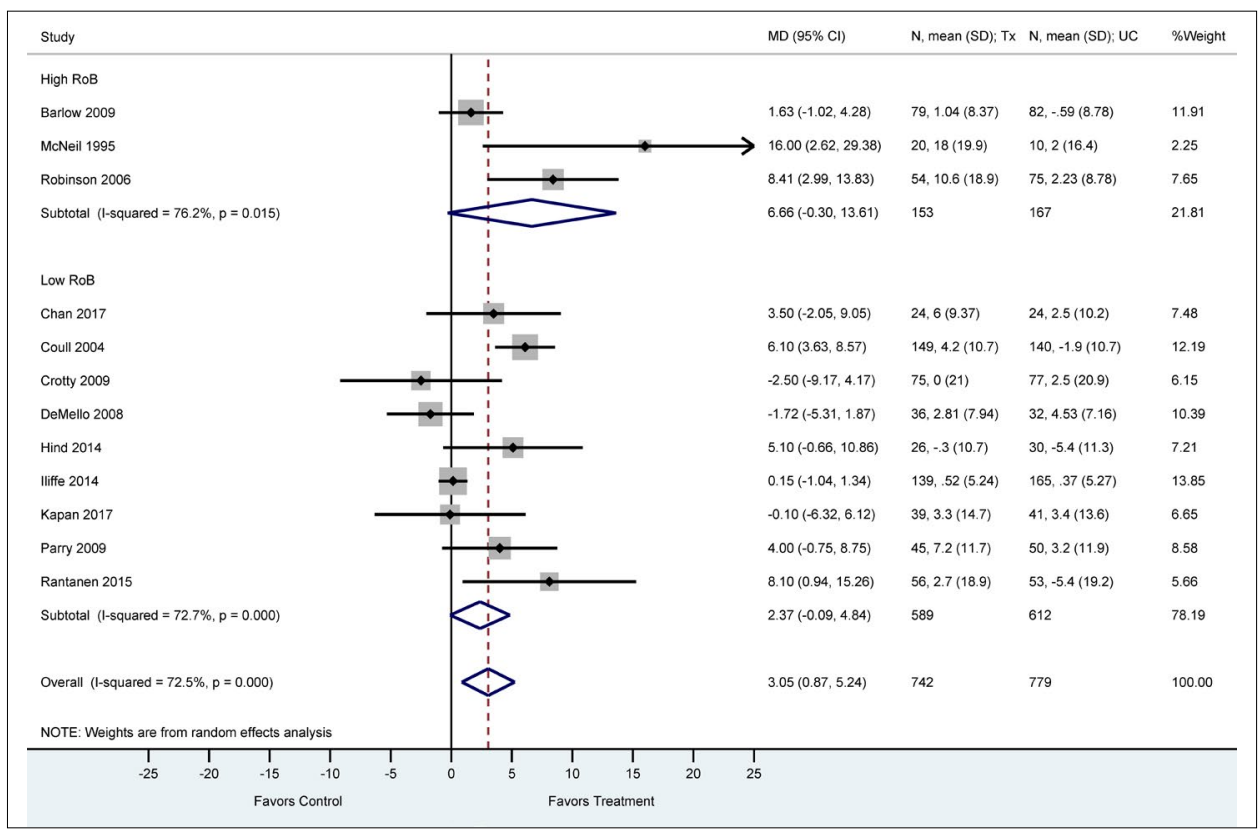


MOORE: VOLUNTEER IMPACT ON SENIORS

D.2 Physical function (subgroup analysis—outcome assessors adequately blinded)

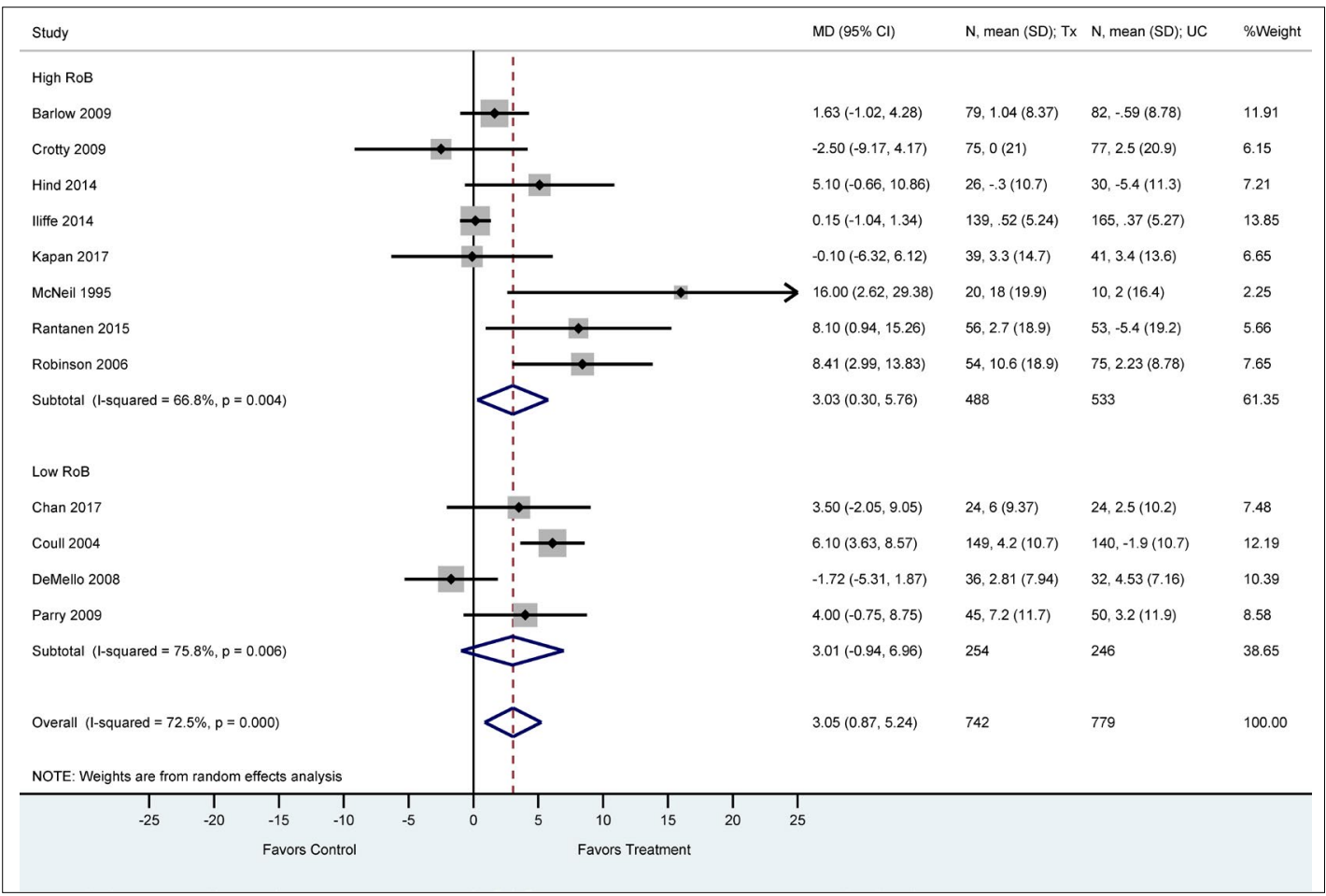

D.3 Physical function (subgroup analysis - incomplete reporting, $>20 \%$, missing participant data)

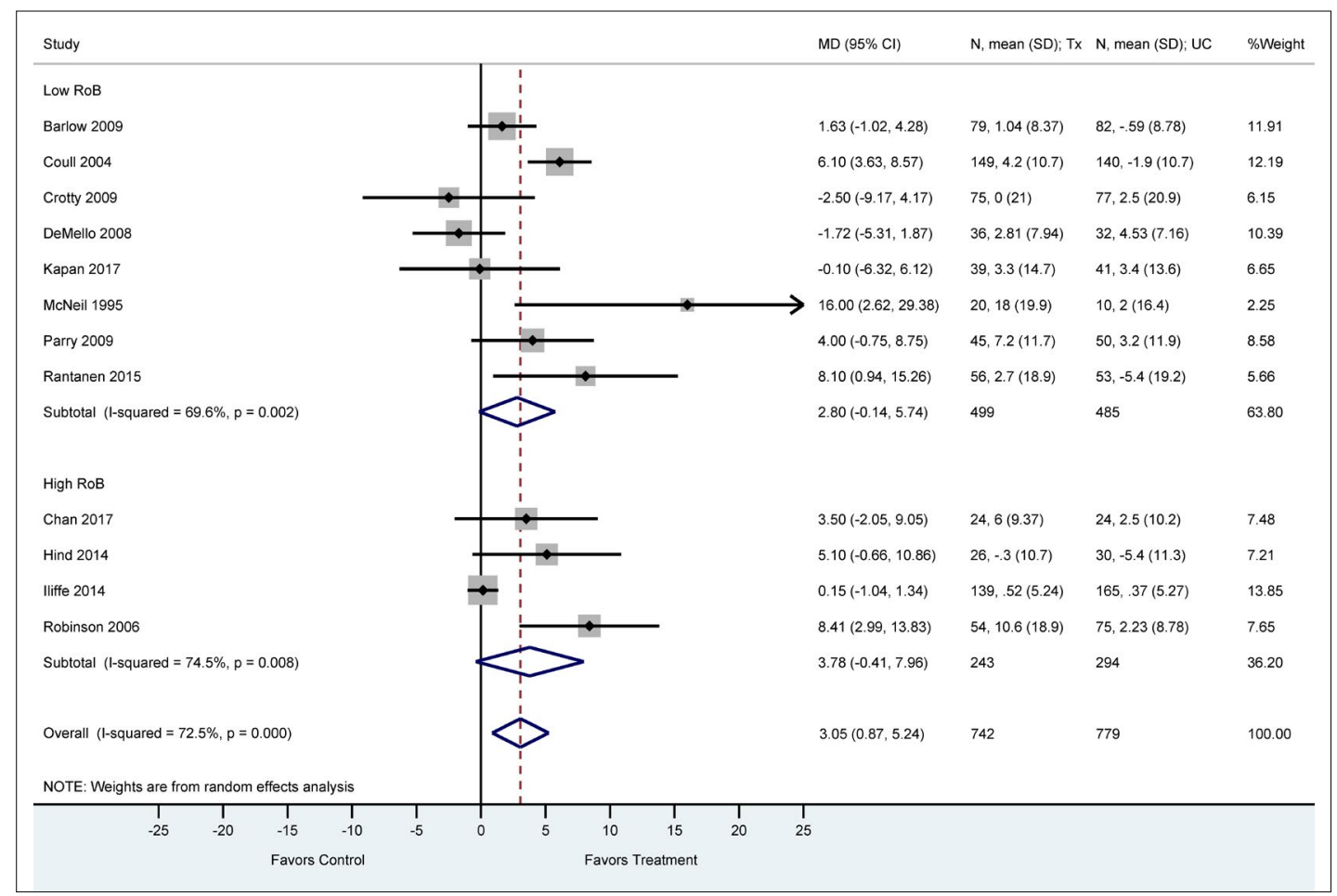


MOORE: VOLUNTEER IMPACT ON SENIORS

D.4 Physical function (sensitivity analysis — excluding studies with imputed SD)

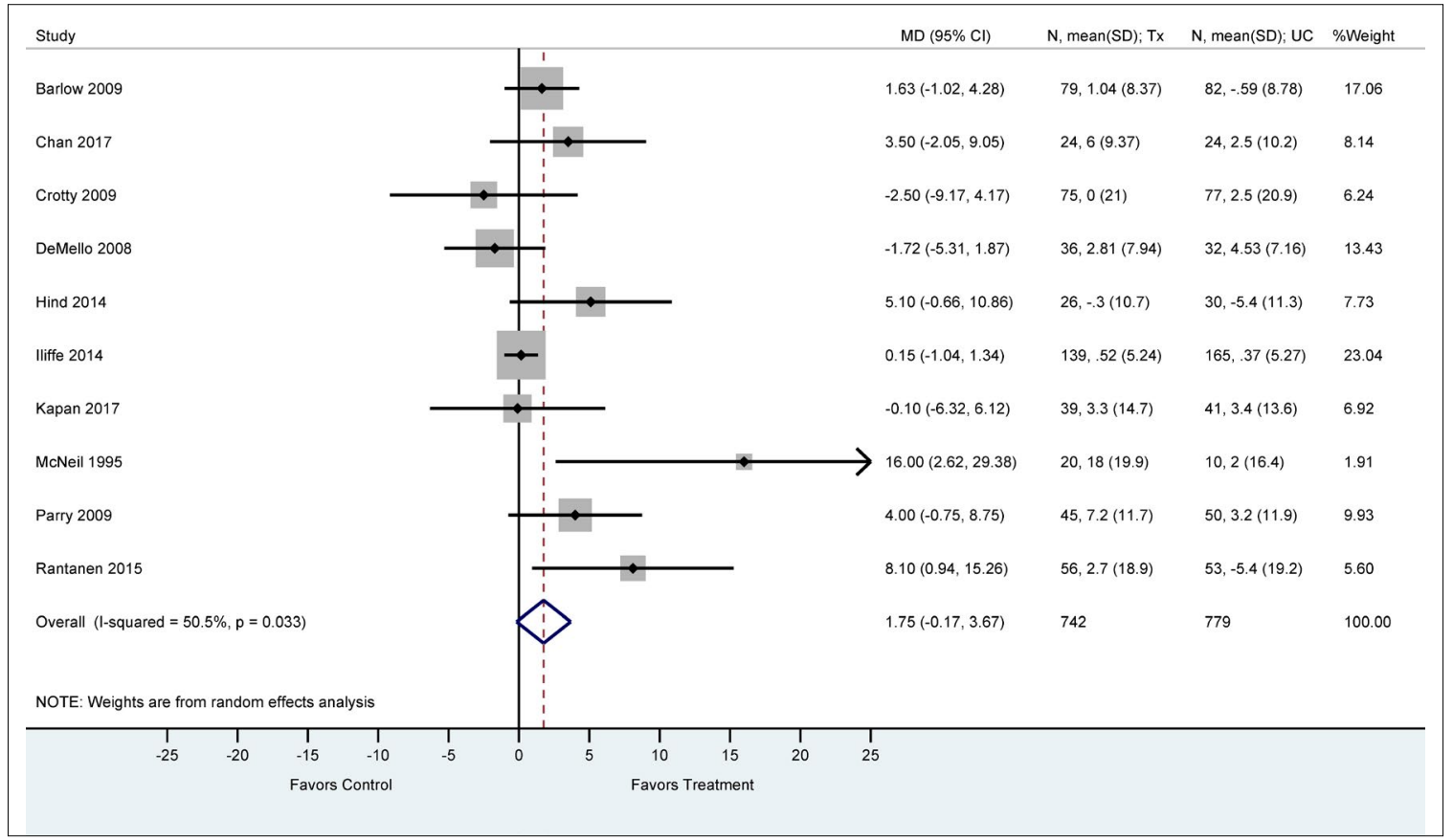

D.5 Physical function funnel plot (small study effect not significant; $p$ value for Egger's test $=.06$ ); meta-regression: no covariates (physical health) explained observed heterogeneity

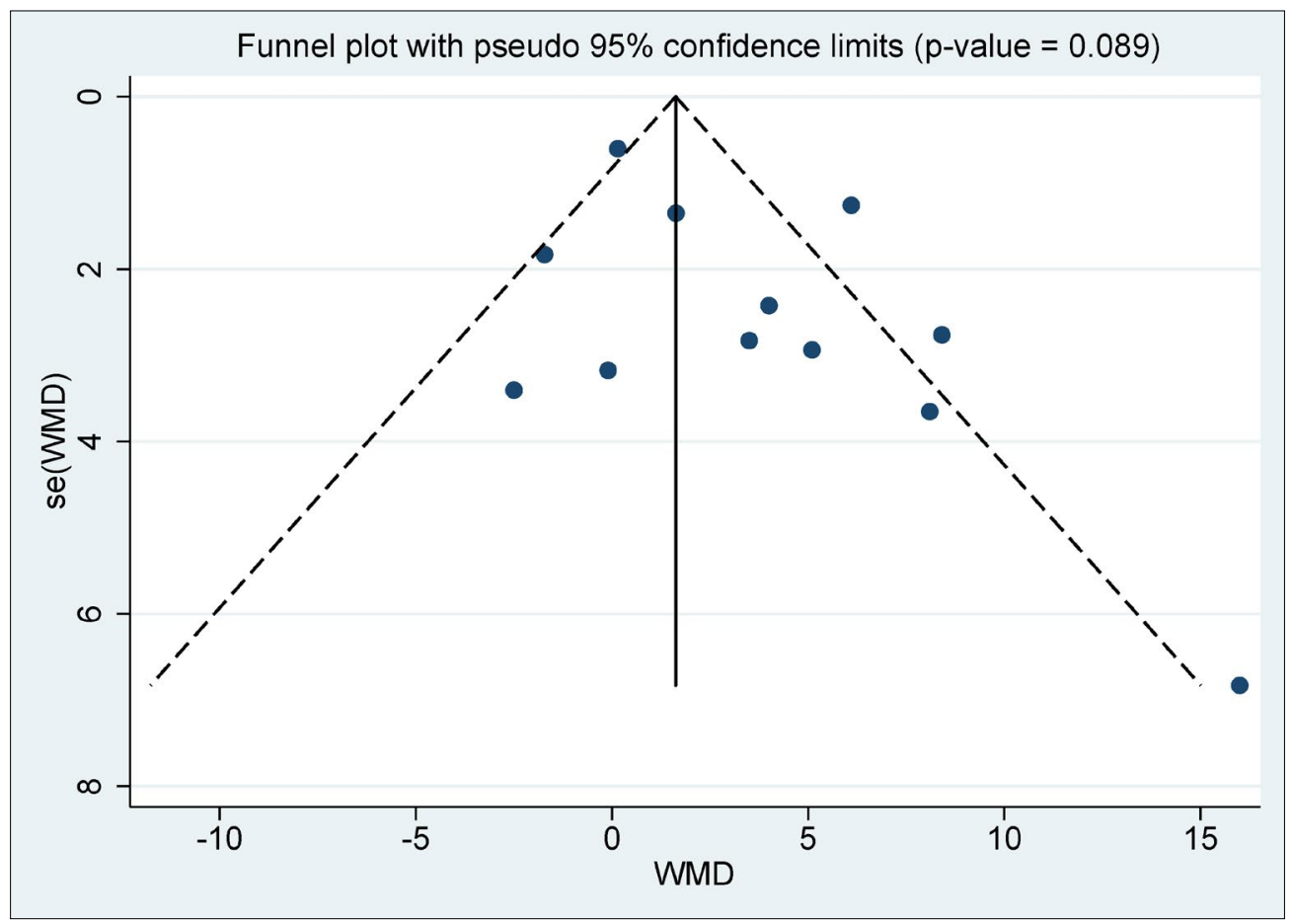


MOORE: VOLUNTEER IMPACT ON SENIORS

APPENDIX E: Physical activity (standardized mean difference)

E.1 Physical activity (subgroup analysis—adequately concealed allocation)

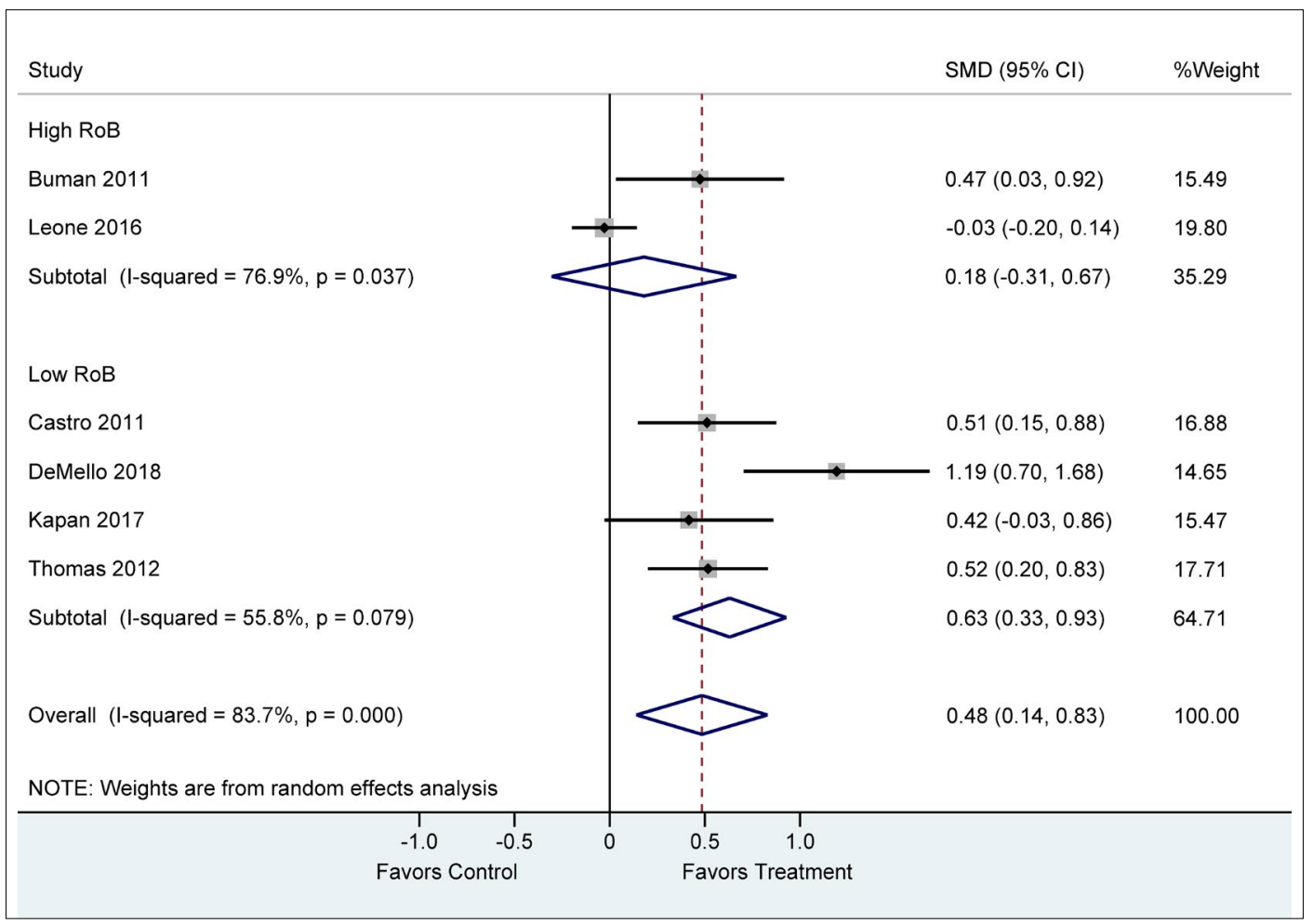

E.2 Physical activity (subgroup analysis — outcome assessors blinded); analyses for incomplete outcome reporting and imputed standard deviation not relevant (no studies affected); not enough studies to test for small study effect (publication bias)

\begin{tabular}{|c|c|c|c|}
\hline Study & & $\operatorname{SMD}(95 \% \mathrm{Cl})$ & \%Weight \\
\hline \multicolumn{4}{|l|}{ High RoB } \\
\hline Buman 2011 & $\rightarrow$ & $0.47(0.03,0.92)$ & 15.49 \\
\hline DeMello 2018 & $\rightarrow$ & $1.19(0.70,1.68)$ & 14.65 \\
\hline Leone 2016 & & $-0.03(-0.20,0.14)$ & 19.80 \\
\hline Thomas 2012 & - & $0.52(0.20,0.83)$ & 17.71 \\
\hline Subtotal $(I-$ squared $=89.3 \%, p=0.000)$ & & $0.51(0.01,1.01)$ & 67.65 \\
\hline \multicolumn{4}{|l|}{ Low RoB } \\
\hline Castro 2011 & & $0.51(0.15,0.88)$ & 16.88 \\
\hline Kapan 2017 & & $0.42(-0.03,0.86)$ & 15.47 \\
\hline Subtotal $(\mathrm{I}-$ squared $=0.0 \%, p=0.746)$ & & $0.47(0.19,0.75)$ & 32.35 \\
\hline Overall $(I-$ squared $=83.7 \%, p=0.000)$ & & $0.48(0.14,0.83)$ & 100.00 \\
\hline \multicolumn{4}{|l|}{ NOTE: Weights are from random effects analysis } \\
\hline $\begin{array}{cc} & \\
-1.0 & -0.5\end{array}$ & 1.0 & & \\
\hline Favors Control & Favors Treatment & & \\
\hline
\end{tabular}


MOORE: VOLUNTEER IMPACT ON SENIORS

\section{APPENDIX F: Emotional function (mean difference SF-36 mental component score-100 point scale)}

F.1 Emotional function (subgroup analysis - adequately concealed allocation)

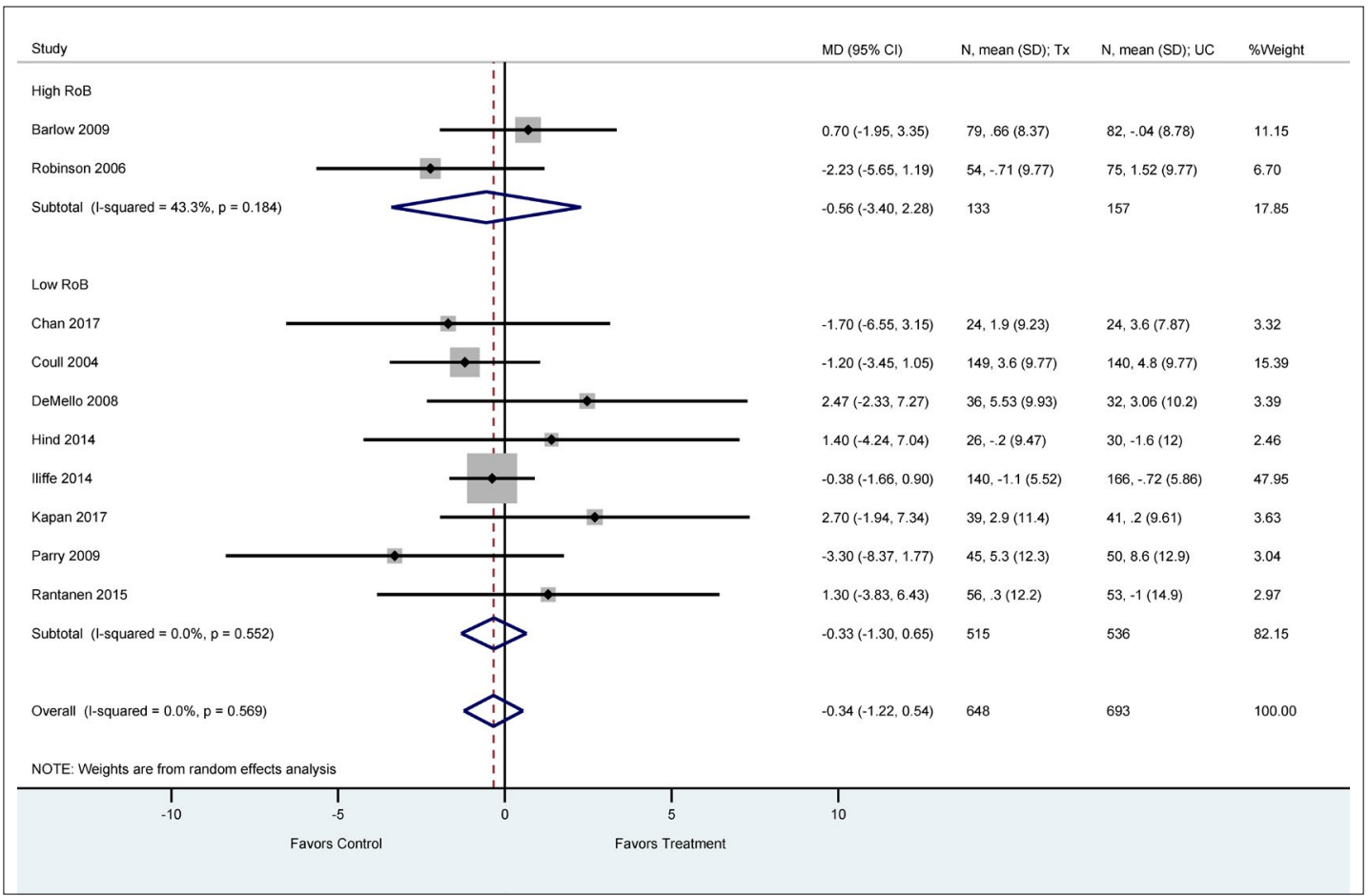

F.2 Emotional function (subgroup analysis—outcome assessor adequately blinded)

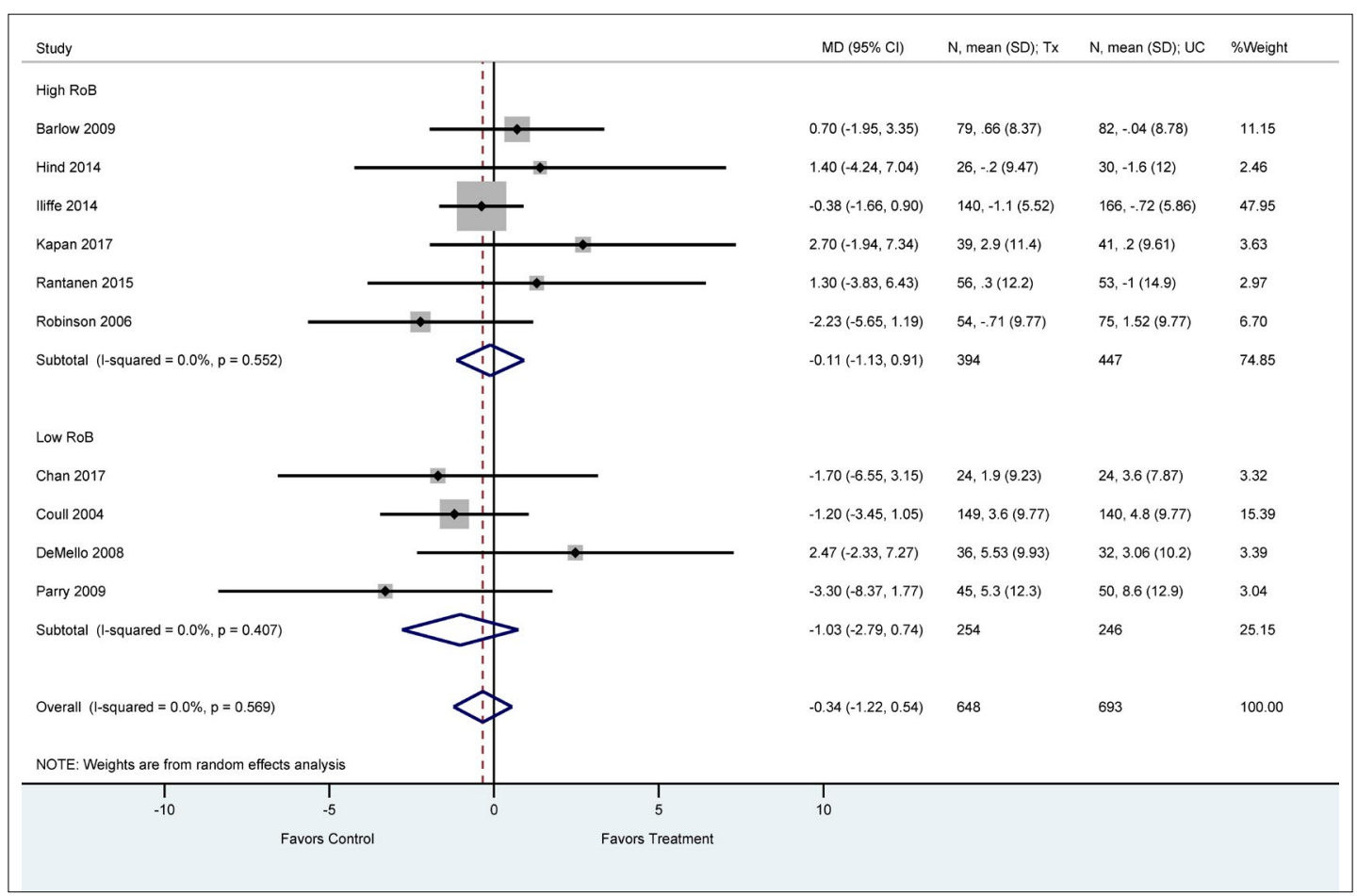


MOORE: VOLUNTEER IMPACT ON SENIORS

F.3 Emotional function (subgroup analysis - incomplete reporting $>20 \%$ missing participant data)

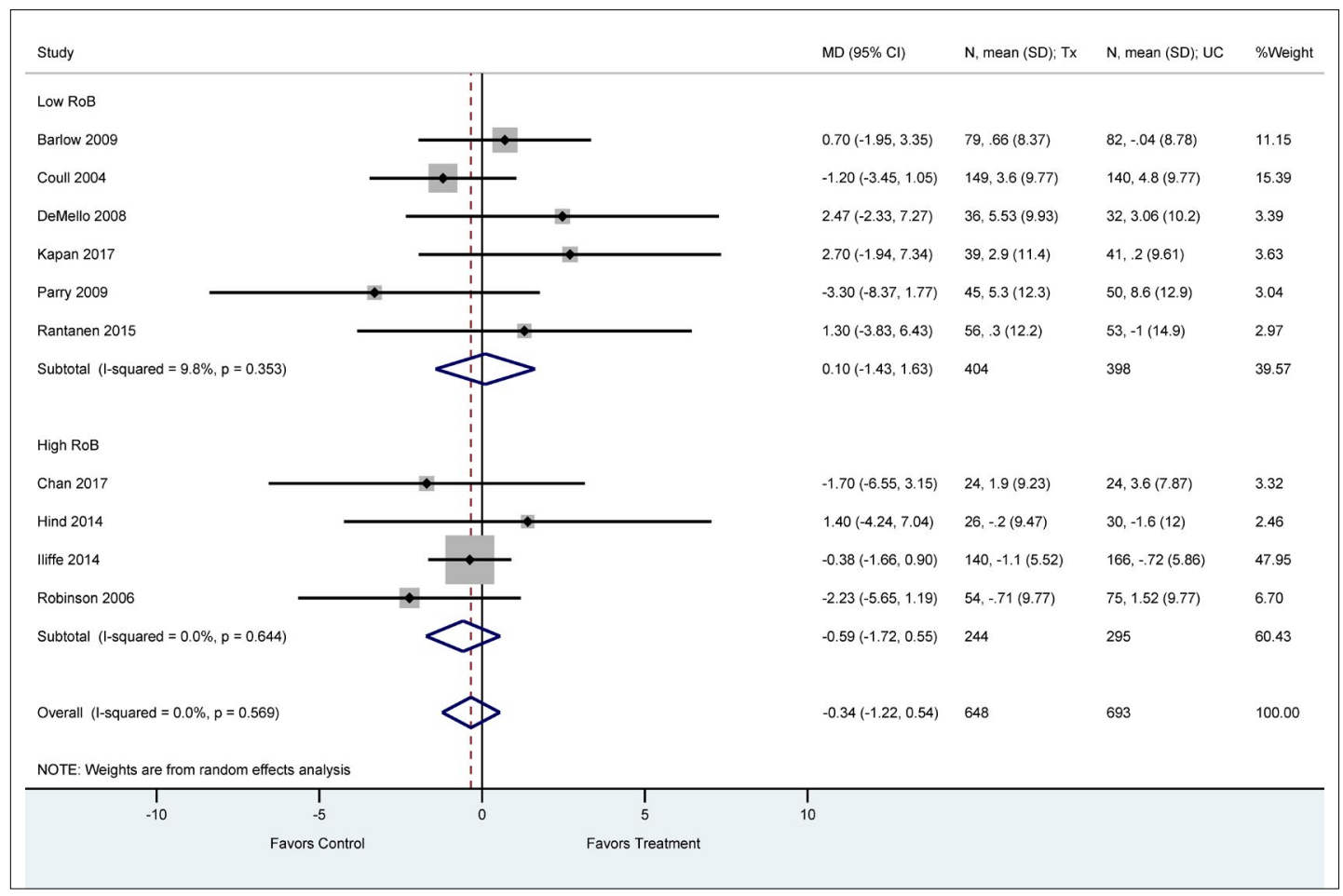

F.4 Emotional function (subgroup analysis - excluding studies with imputed standard deviation)

\begin{tabular}{|c|c|c|c|c|c|}
\hline Study & & $\operatorname{MD}(95 \% \mathrm{Cl})$ & $N$, mean $(S D) ; T x$ & $\mathrm{~N}$, mean (SD); UC & $\%$ Weight \\
\hline Barlow 2009 & - & $0.70(-1.95,3.35)$ & $79, .66(8.37)$ & $82,-.04(8.78)$ & 14.31 \\
\hline Chan 2017 & & $-1.70(-6.55,3.15)$ & $24,1.9(9.23)$ & $24,3.6(7.87)$ & 4.26 \\
\hline DeMello 2008 & $\rightarrow$ & $2.47(-2.33,7.27)$ & $36,5.53(9.93)$ & $32,3.06(10.2)$ & 4.35 \\
\hline Hind 2014 & 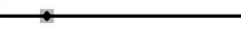 & $1.40(-4.24,7.04)$ & $26,-.2(9.47)$ & 30, $-1.6(12)$ & 3.16 \\
\hline Iliffe 2014 & & $-0.38(-1.66,0.90)$ & $140,-1.1(5.52)$ & $166,-.72(5.86)$ & 61.54 \\
\hline Kapan 2017 & $\rightarrow$ & $2.70(-1.94,7.34)$ & $39,2.9(11.4)$ & $41, .2(9.61)$ & 4.66 \\
\hline Parry 2009 & & $-3.30(-8.37,1.77)$ & $45,5.3(12.3)$ & $50,8.6(12.9)$ & 3.91 \\
\hline Rantanen 2015 & - & $1.30(-3.83,6.43)$ & $56.3(12.2)$ & 53, $-1(14.9)$ & 3.81 \\
\hline Overall (I-squared $=0.0 \%, p=0.599$ ) & & $-0.01(-1.01,0.99)$ & 648 & 693 & 100.00 \\
\hline NOTE: Weights are from random effects analysis & & & & & \\
\hline$\underset{-10}{T}$ & $\begin{array}{l}1 \\
5\end{array}$ & $\begin{array}{l}T \\
10\end{array}$ & & & \\
\hline Favors Control & Favors Treatment & & & & \\
\hline
\end{tabular}


F.5 Emotional function funnel plot (small study effect not significant; $p$ value for Egger's test $=.589$ )

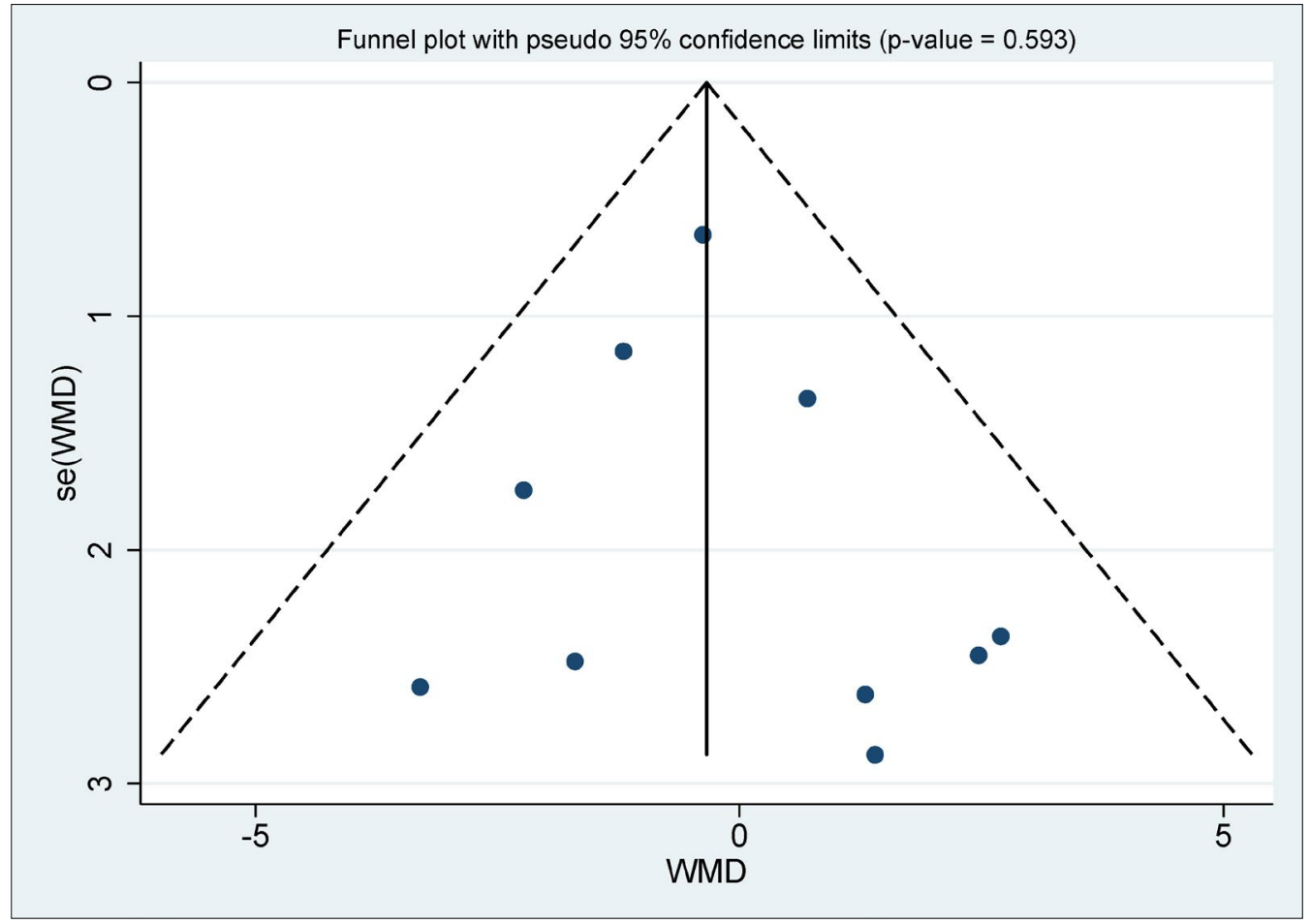

APPENDIX G: Depression (mean difference HADS-10 point scale)

G.1 Depression (subgroup analysis - adequately concealed allocation)

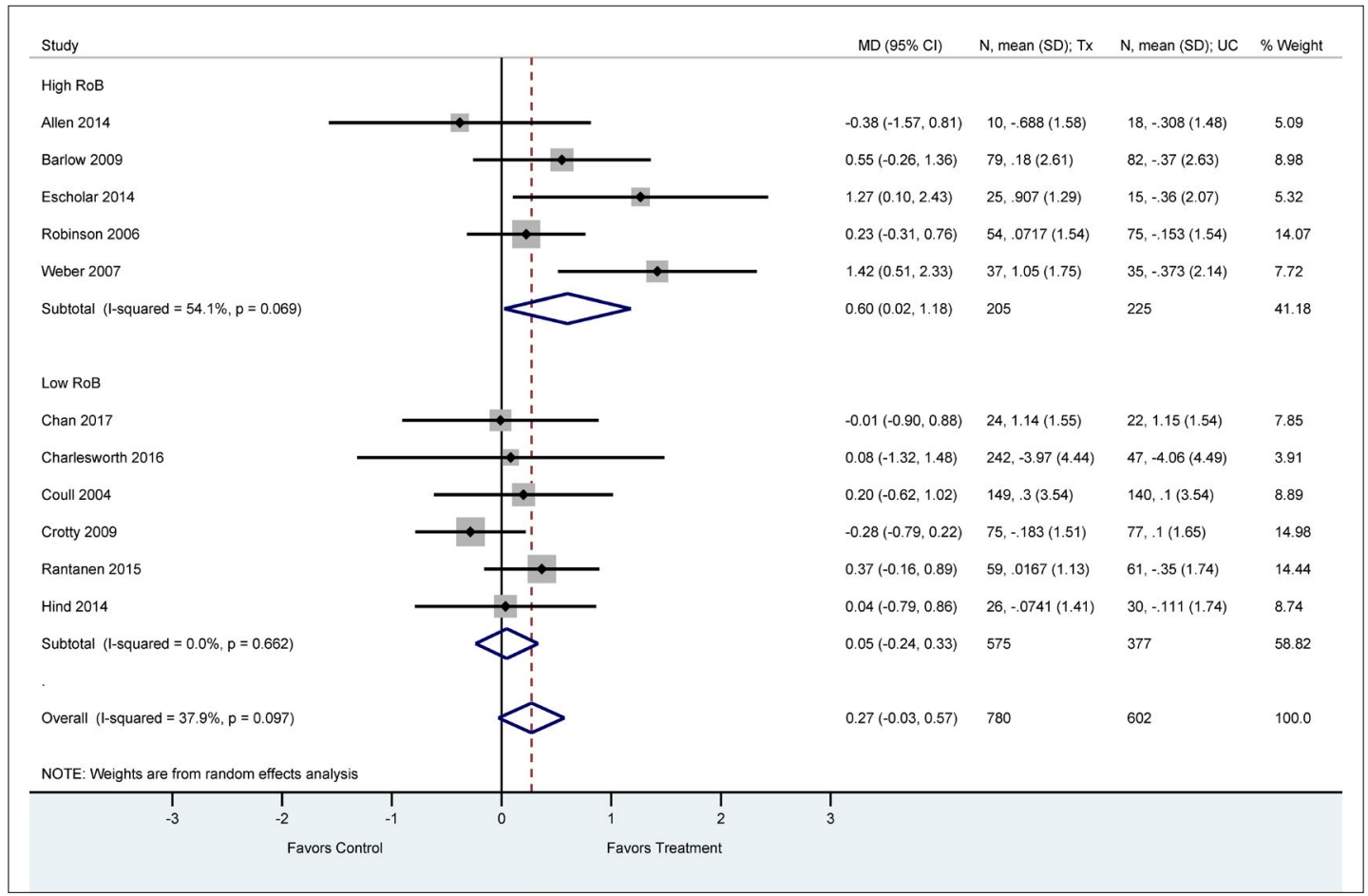


MOORE: VOLUNTEER IMPACT ON SENIORS

G.2 Depression (subgroup analysis — outcome assessors adequately blinded)

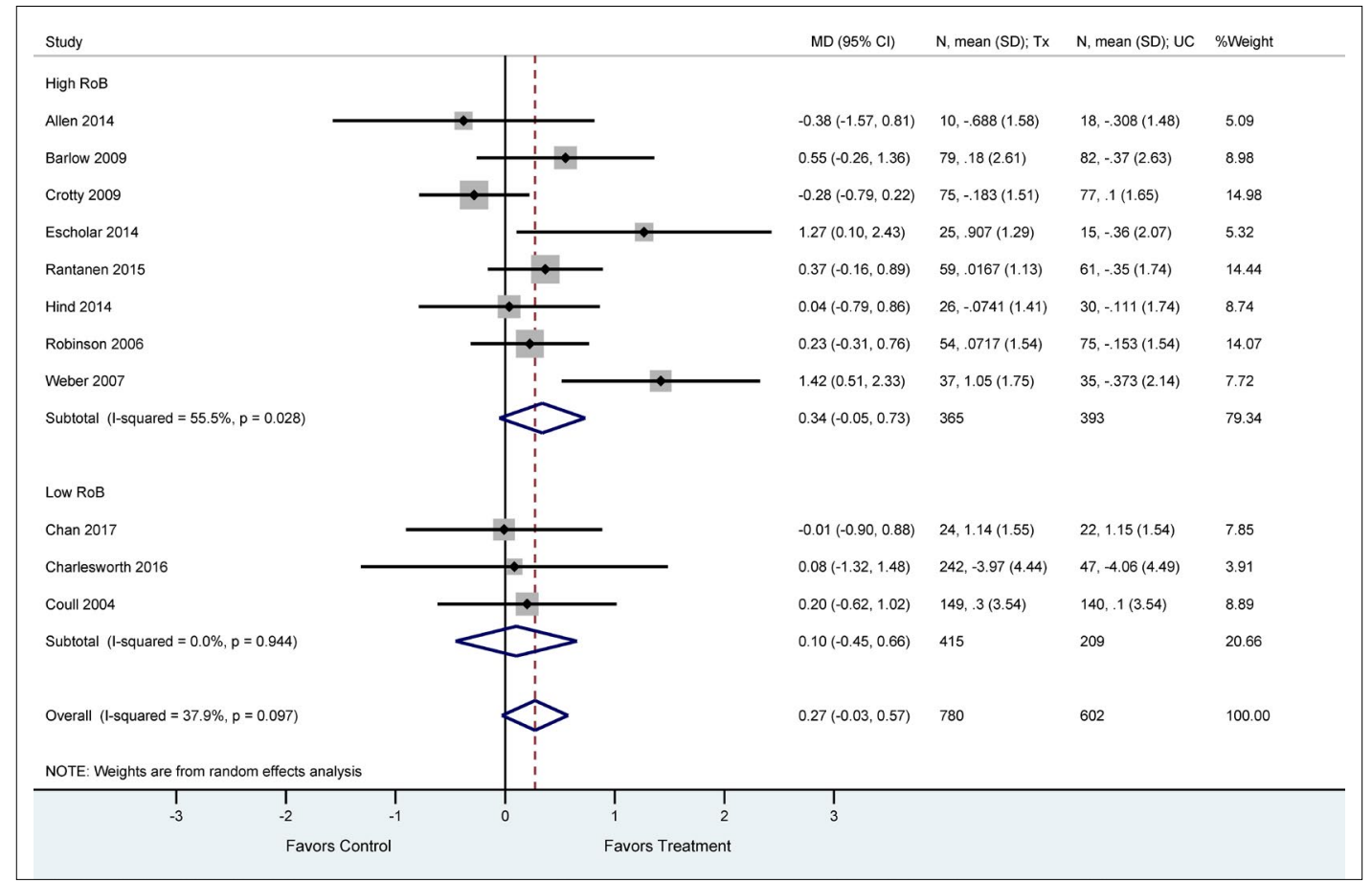

G.3 Depression (subgroup analysis - incomplete reporting $>20 \%$ missing participant data)

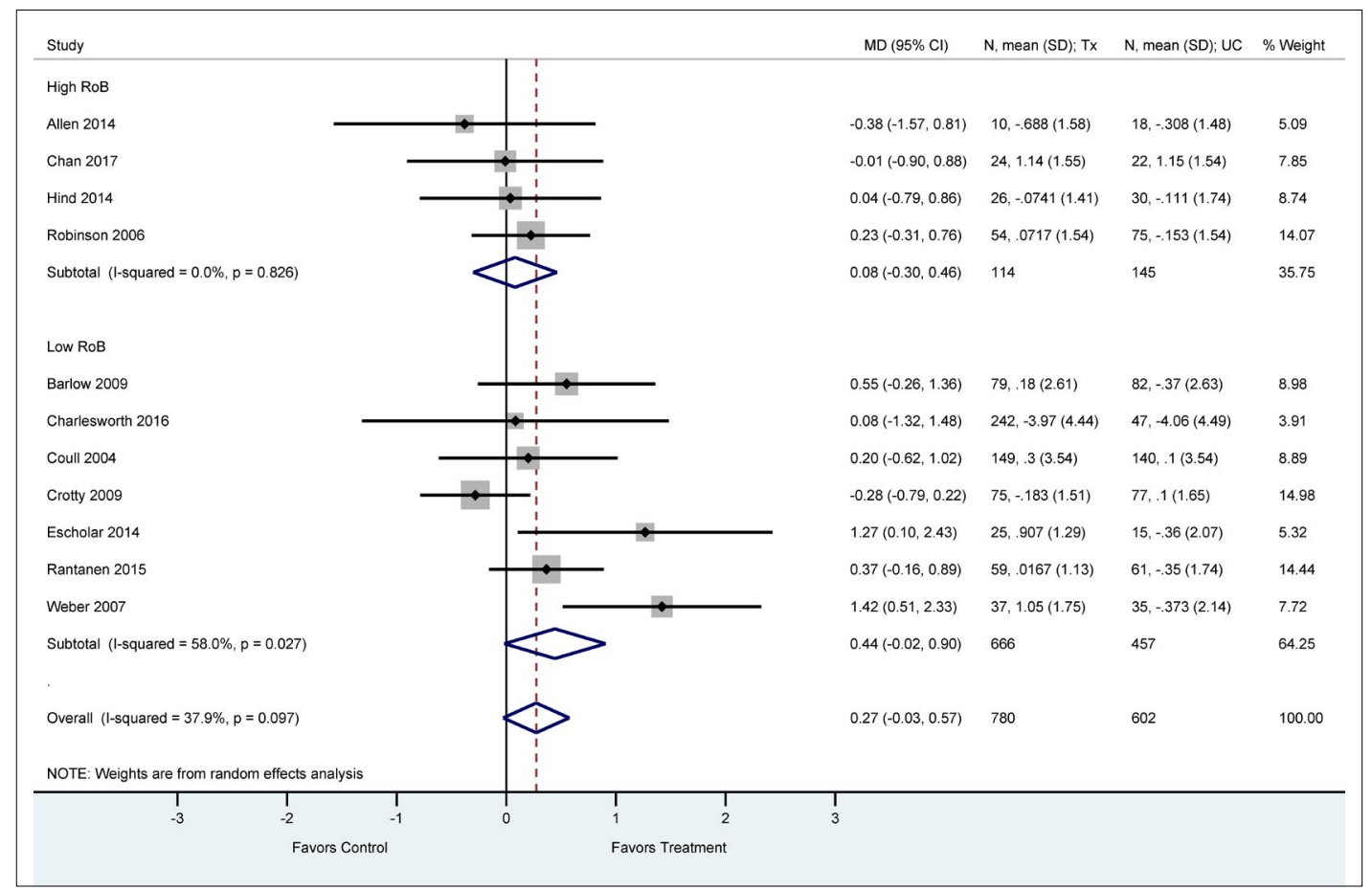


MOORE: VOLUNTEER IMPACT ON SENIORS

G.4 Depression (sensitivity analysis - excluding studies with imputed standard deviation)

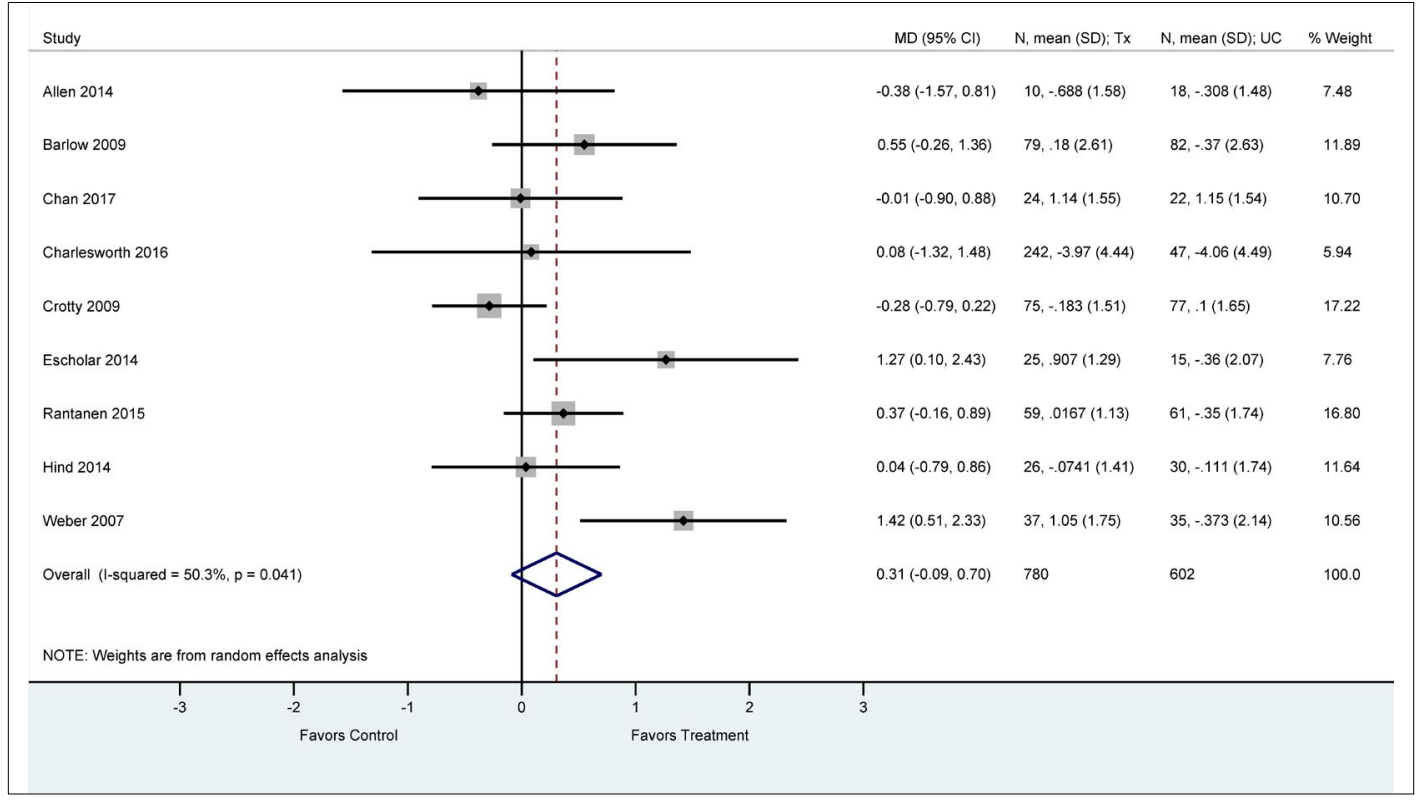

G.5 Depression funnel plot (small study effect not significant; $p$ value for Egger's test $=.356$ )

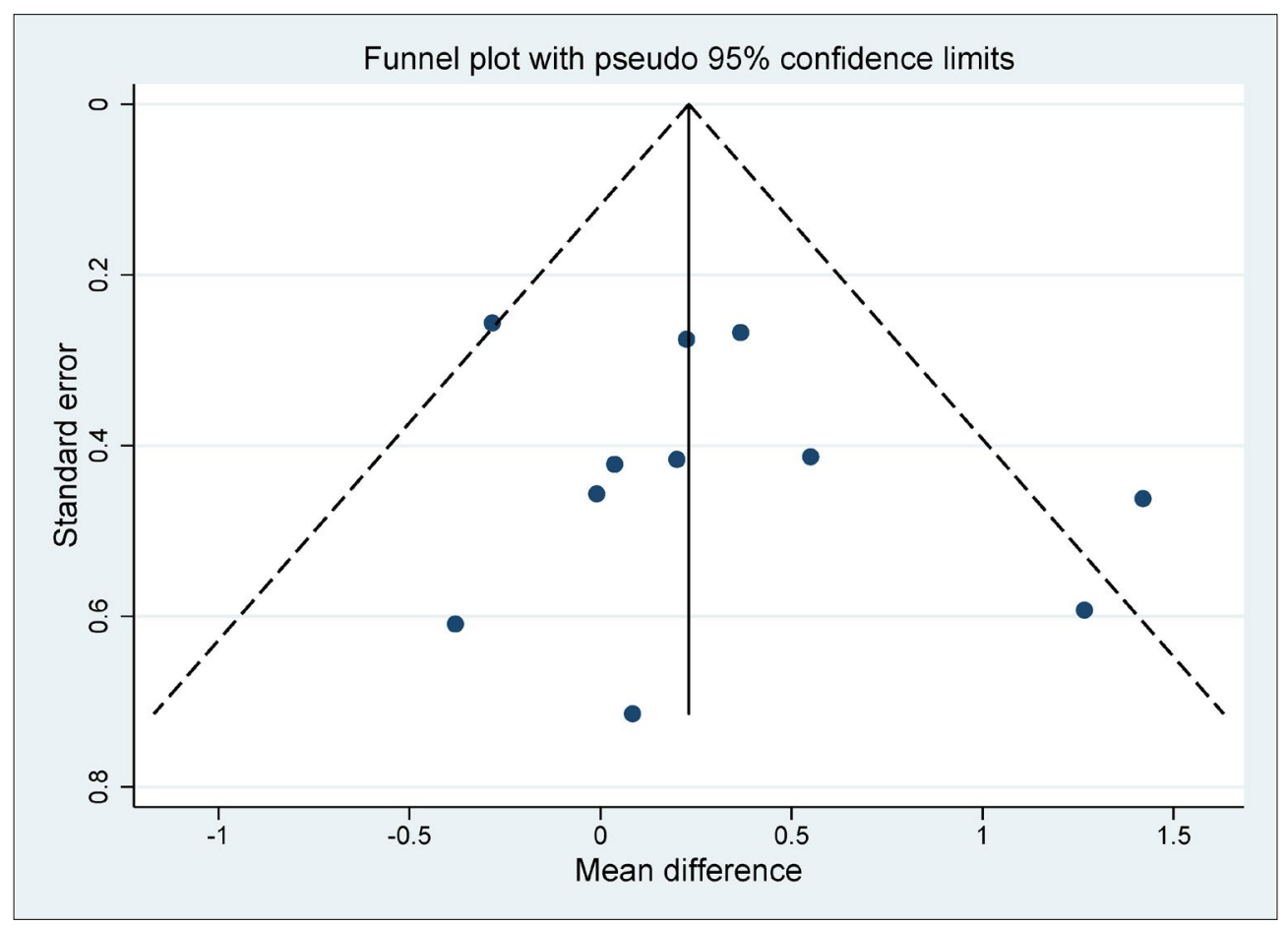


MOORE: VOLUNTEER IMPACT ON SENIORS

APPENDIX H: Anxiety (mean difference HADS-10 point scale)

H.1 Anxiety (subgroup analysis - adequately concealed allocation)

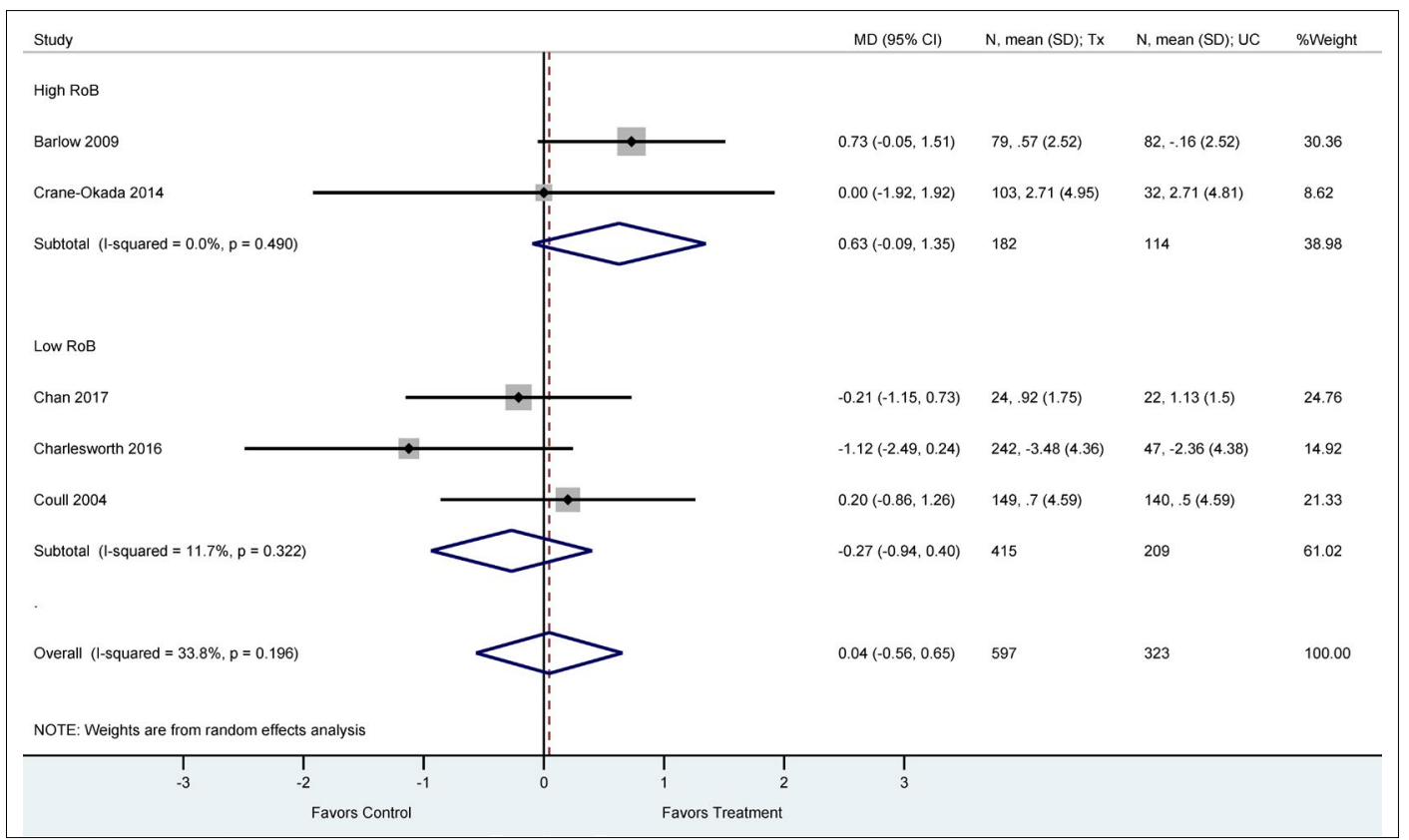

H.2 Anxiety (subgroup analysis — outcome assessors adequately blinded)

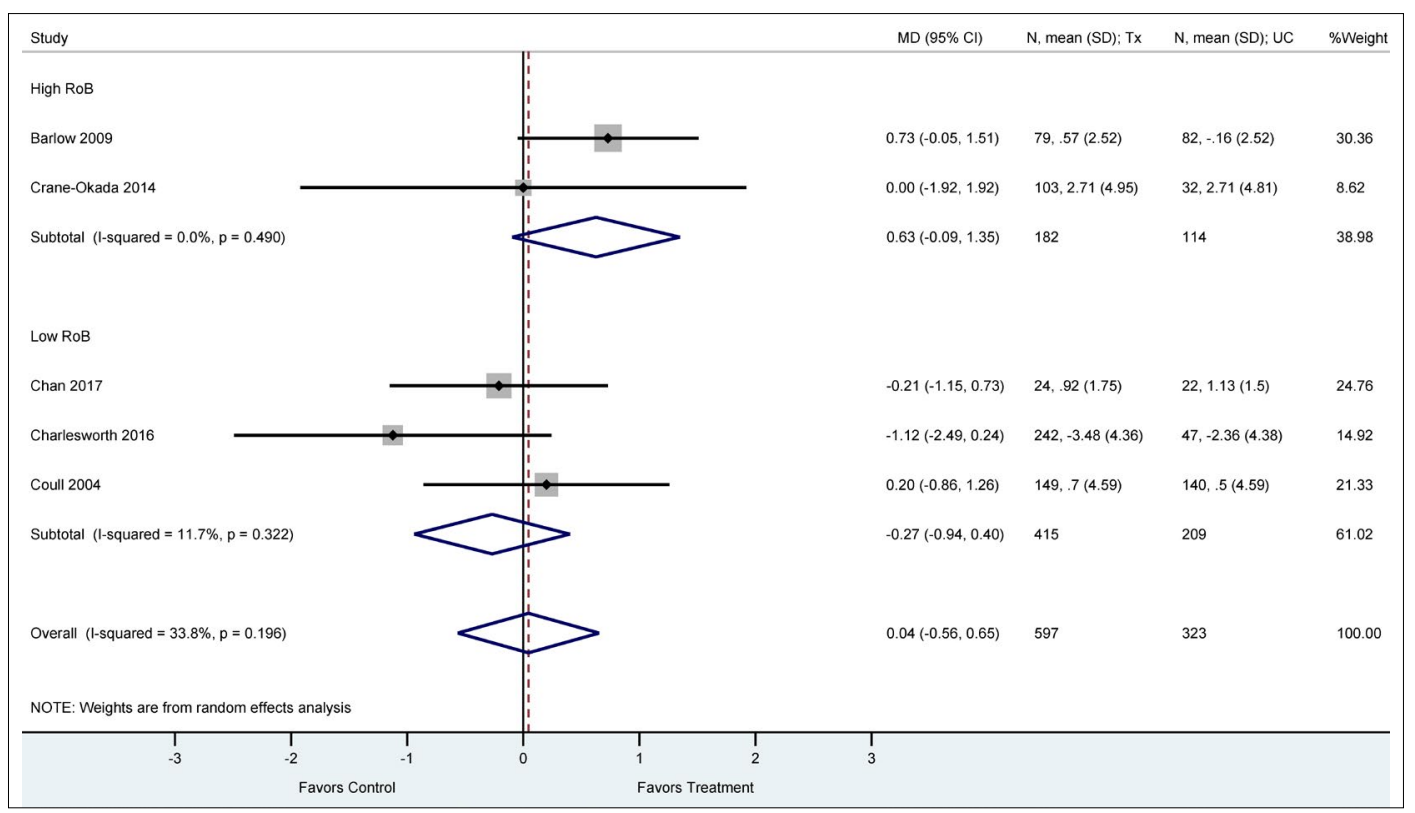


MOORE: VOLUNTEER IMPACT ON SENIORS

H.3 Anxiety (sensitivity analysis—excluding studies with imputed standard deviation)

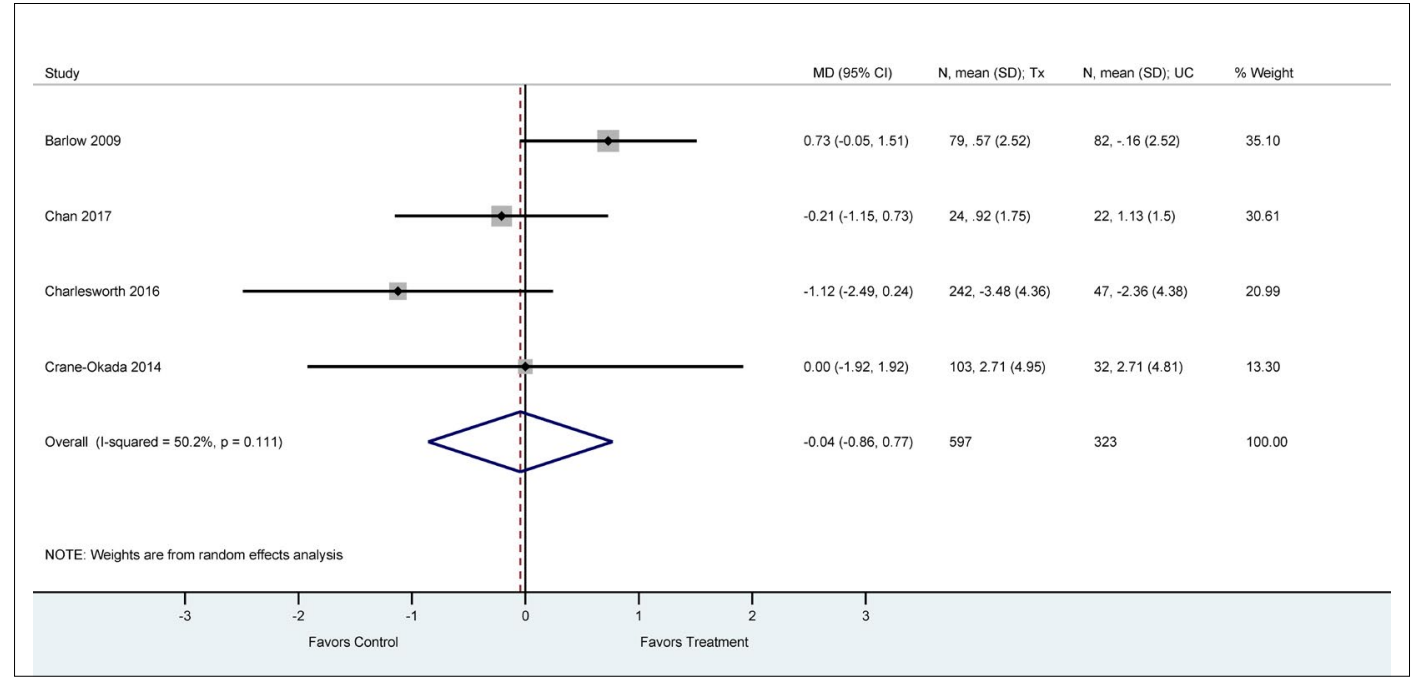

APPENDIX I: Quality of life (EQ 5D; 0-1 point scale)

I.1 Quality of life (subgroup analysis—adequately concealed allocation)

\begin{tabular}{|c|c|c|c|c|c|}
\hline Study & & MD $(95 \% \mathrm{Cl})$ & $\mathrm{N}$, mean (SD); $\mathrm{Tx}$ & $\mathrm{N}$, mean (SD); UC & $\%$ Weight \\
\hline \multicolumn{6}{|l|}{ Low RoB } \\
\hline Charlesworth 2016 & - & $0.05(-0.06,0.15)$ & $242, .124(.339)$ & $47, .077(.339)$ & 1.84 \\
\hline Crotty 2009 & & $0.05(-0.03,0.13)$ & $75,-.01(.256)$ & 77, -.06 (.236) & 3.34 \\
\hline Iliffe 2014 & & $-0.01(-0.03,0.01)$ & $134,0(.0819)$ & $162, .01(.0755)$ & 62.88 \\
\hline Johansson 2016 & & $-0.03(-0.08,0.02)$ & $108,-.03(.183)$ & $125,0(.19)$ & 8.93 \\
\hline Kapan 2017 & & $0.03(-0.03,0.09)$ & $39, .056(.149)$ & $41, .025(.134)$ & 5.31 \\
\hline Rantanen 2015 & & $0.02(-0.03,0.06)$ & $56, .004(.118)$ & $53,-.014(.131)$ & 9.38 \\
\hline Safford 2015 & & $0.01(-0.04,0.06)$ & $111,-.006(.2)$ & $112,-.017(.2)$ & 7.47 \\
\hline Hind 2014 & 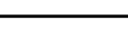 & $0.02(-0.14,0.18)$ & $26,0(.324)$ & $29,-.02(.256)$ & 0.85 \\
\hline Subtotal (I-squared $=0.0 \%, p=0.465$ ) & & $-0.00(-0.02,0.01)$ & 791 & 646 & 100.00 \\
\hline Overall (I-squared $=0.0 \%, p=0.465$ ) & & $-0.00(-0.02,0.01)$ & 791 & 646 & 100.00 \\
\hline \multicolumn{6}{|l|}{ NOTE: Weights are from random effects analysis } \\
\hline${ }_{-0.1}^{1}$ & $T_{0.1}^{1}$ & $\begin{array}{c}1 \\
0.2\end{array}$ & & & \\
\hline Favors Control & Favors Treatment & & & & \\
\hline
\end{tabular}


MOORE: VOLUNTEER IMPACT ON SENIORS

I.2 Quality of life (subgroup analysis—outcome assessors adequately blinded)

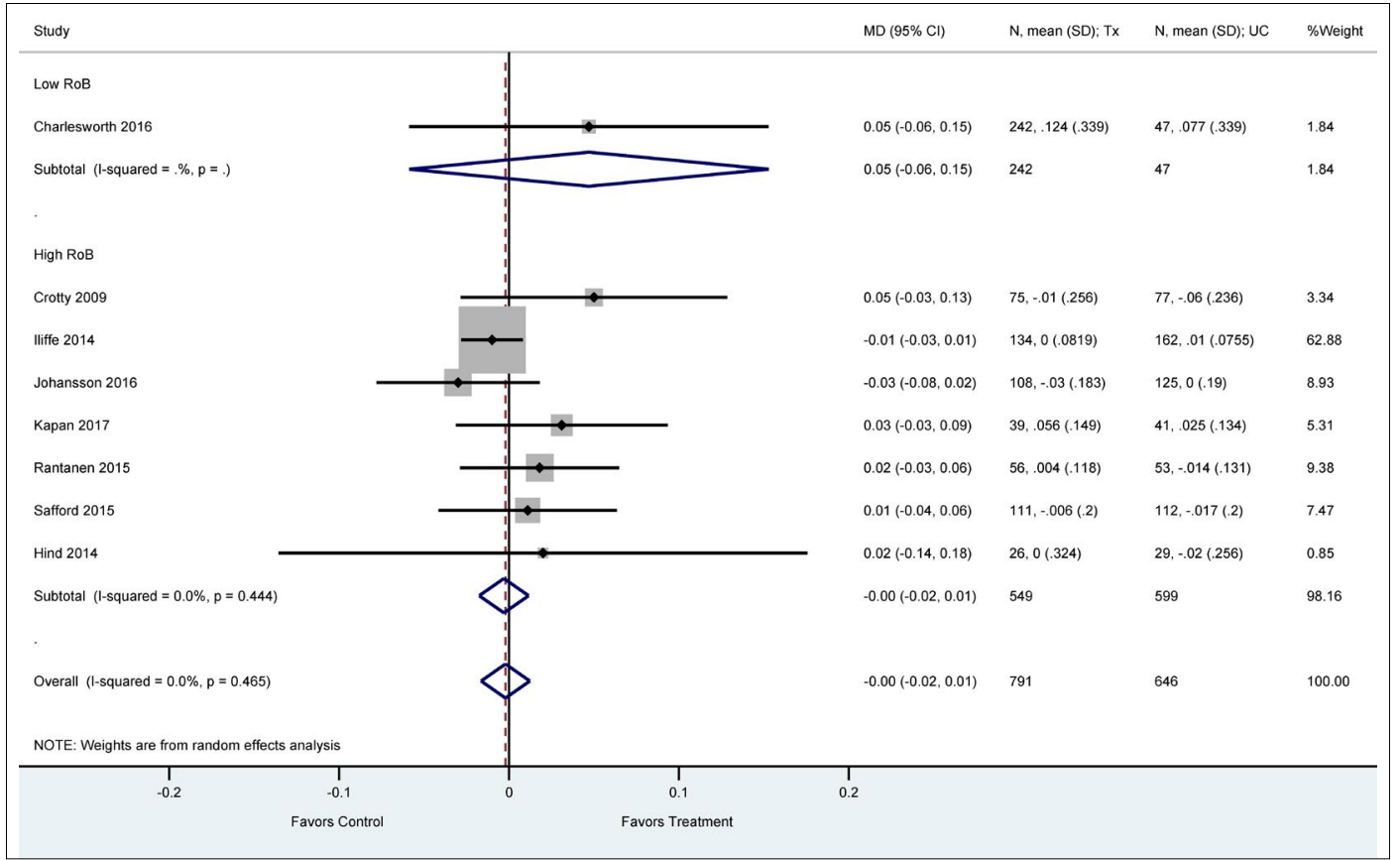

I.3 Quality of life (subgroup analysis - incomplete reporting $>20 \%$ missing participant data)

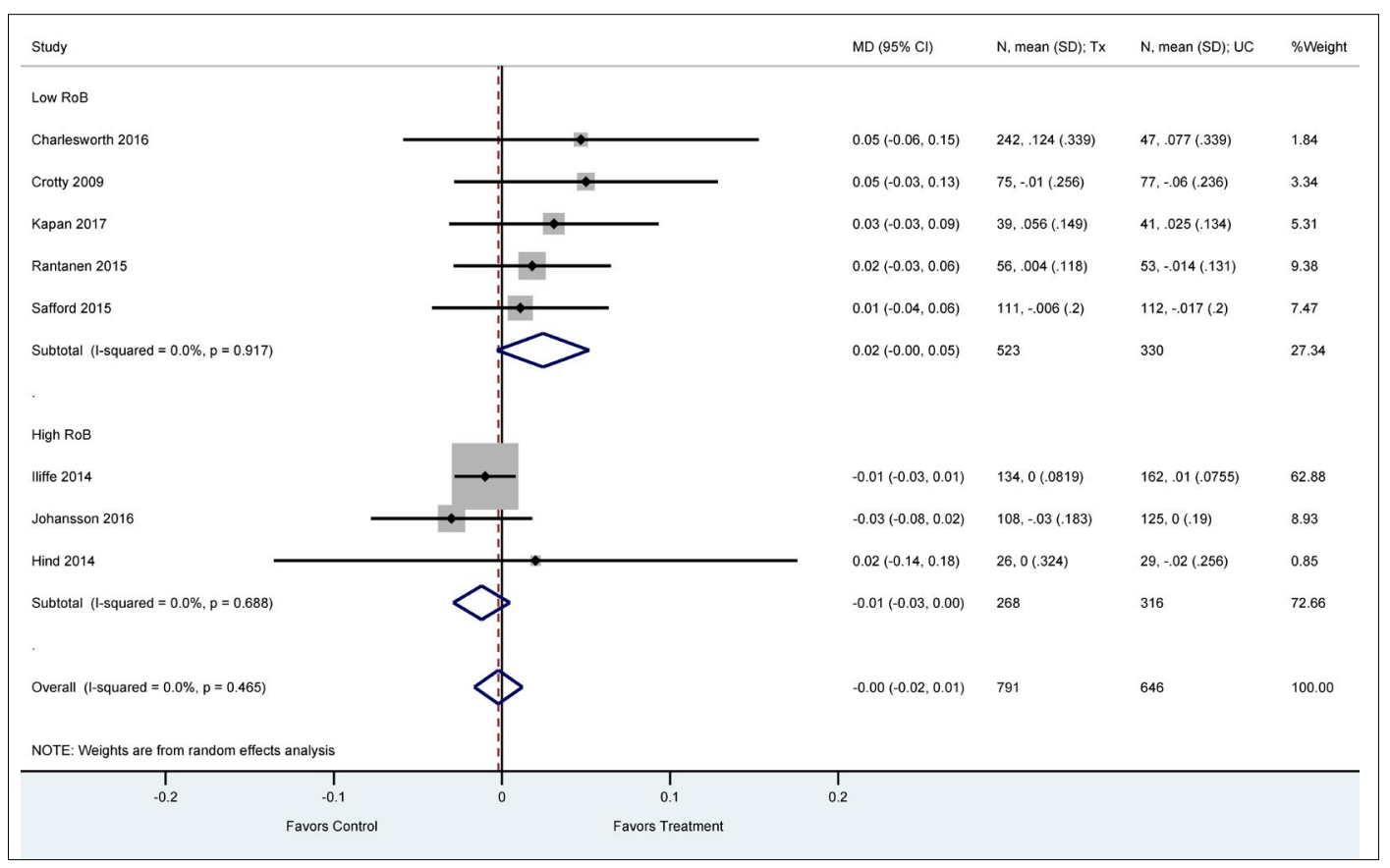


I.4 Quality of life funnel plot (small study effect significant; $p$ value Egger's test $=.054$ )

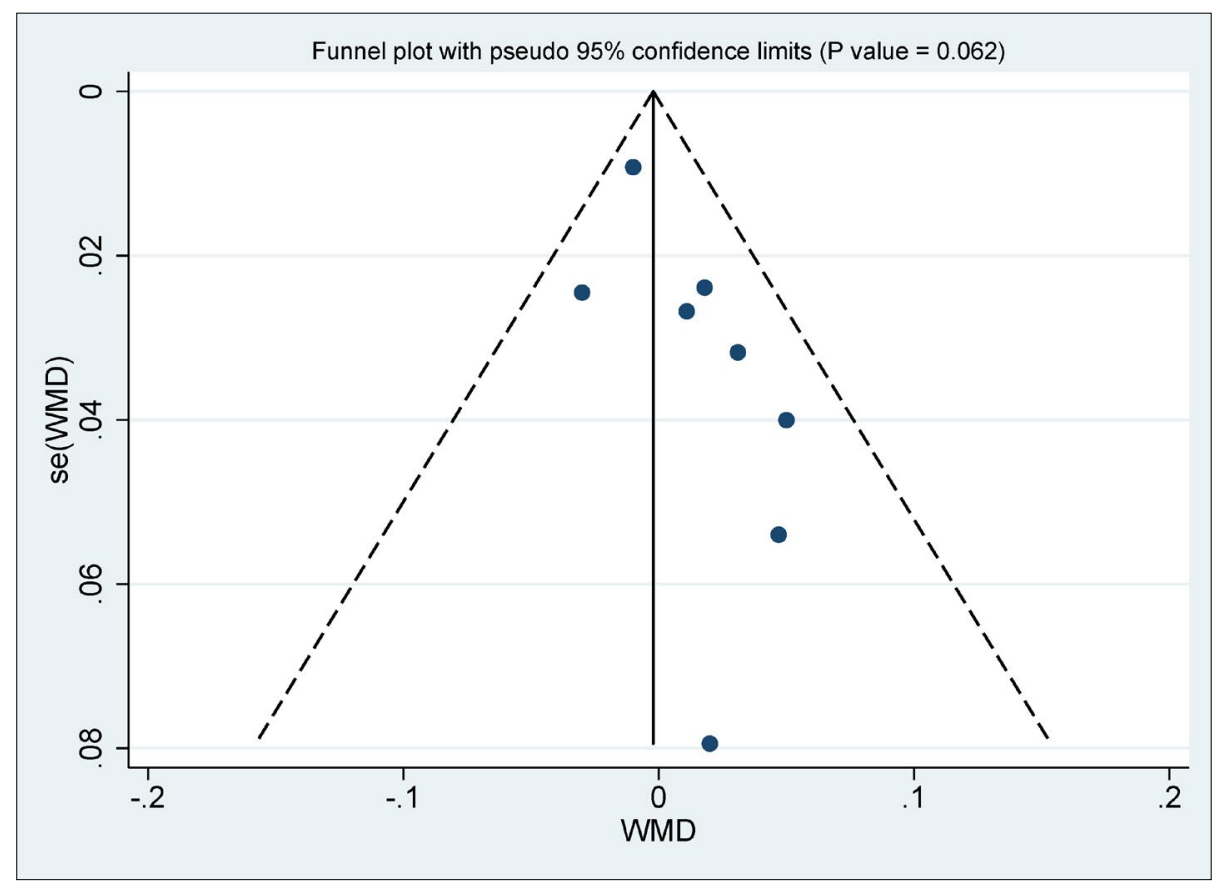

\section{REFERENCES}

1. World Health Organization. Global strategy and action plan on ageing and health. Geneva, Switzerland: World Health Organization; 2017. License: CC BY-NC-SA 3.0 IGO. Available from: https://www.who.int/ageing/WHO-GSAP-2017.pdf

2. United Nations DoEaSA, Population Division. World population prospects: comprehensive tables (ST/ESA/SER.A/399). New York: United Nations; 2017. Available from: https:// population.un.org/wpp/Publications/Files/WPP2017_VolumeI_Comprehensive-Tables.pdf

3. Gilburt H, Buck D, Smith, J. Volunteering in general practice: opportunities and insights. London, UK: The King's Fund; 2018.

4. Dorgo S, King GA, Bader JO, et al. Comparing the effectiveness of peer mentoring and student mentoring in a 35-week fitness program for older adults. Arch Gerontol Geriatr. 2011;52(3):344-49. 5. Rantakokko M, Pakkala I, Äyräväinen I, et al. The effect of out-of-home activity intervention delivered by volunteers on depressive symptoms among older people with severe mobility limitations: a randomized controlled trial. Aging Ment Health. 2015;19(3):231-38.

6. Dorgo S, Robinson KM, Bader J. The effectiveness of a peer-mentored older adult fitness program on perceived physical, mental, and social function. J Am Acad Nurse Prac. 2009;21(2):116-22.

7. Luger E, Dorner TE, Haider S, et al. Effects of a home-based and volunteer-administered physical training, nutritional, and social support program on malnutrition and frailty in older persons: a randomized controlled trial. J Am Med Dir Assoc. 2016;17(7):671.e9-.e16.

8. Dale J, Caramlau IO, Lindenmeyer A, at al. Peer support telephone calls for improving health. Cochrane Db Syst Rev. 2008(4).

9. Meyer A, Coroiu A, Korner A. One-to-one peer support in cancer care: a review of scholarship published between 2007 and 2014. Eur J Cancer Care. 2015;24(3):299-312.

10. Avery A, Bostock L, McCullough F. A systematic review investigating interventions that can help reduce consumption of sugar-sweetened beverages in children leading to changes in body fatness. J Hum Nutr Diet. 2015;28(Suppl 1):52-64. Epub $09 / 19$.

11. Lewin S, Dick J, Pond P, et al. Lay health workers in primary and community health care. Cochrane Db Syst Rev. 2005(1).

12. Dolovich L, Oliver D, Lamarche L, et al. Combining volunteers and primary care teamwork to support health goals and needs of older adults: a pragmatic randomized controlled trial. CMAJ. 2019;191(18):E491-E500.

13. Rutherford A, Bu F, Dawson A, et al. Literature review to inform the development of Scotland's volunteering outcomes framework: People, communities and places. Edinburgh: Govt. of Scotland; 2019.

14. Ware, JE, Dewey JE, Kosinski M. How to score Version 2 of the SF-36 Health Survey (Standard and Acute forms), 3rd ed. ed. Lincoln, RI: QualityMetric; 2001.

15. Theiler R, Spielberger J, Bischoff HA, et al. Clinical evaluation of the WOMAC 3.0 OA Index in numeric rating scale format using a computerized touch screen version. Osteoarthr Cartilage. 2002;10(6):479-81.

16. Skevington SM, Lotfy M, O'Connell KA. The World Health Organization's WHOQOL-BREF quality of life assessment: psychometric properties and results of the international field trial. A report from the WHOQOL Group. Qual Life Res. 2004;13(2):299-310.

17. Moore SC, Patel AV, Matthews CE, et al. Leisure time physical activity of moderate to vigorous intensity and mortality: a large pooled cohort analysis. PLoS Med. 2012;9(11):e1001335. Epub $11 / 06$. 
18. Jetté M, Sidney K, Blümchen G. Metabolic equivalents (METS) in exercise testing, exercise prescription, and evaluation of functional capacity. Clin Cardiol. 1990;13(8):555-65.

19. Zigmond AS, Snaith RP. The Hospital Anxiety and Depression Scale. Acta Psychiatr Scand. 1983;67(6):361-70.

20. Veit CT, Ware JE. The structure of psychological distress and well-being in general populations. J Consult Clin Psych. 1983;51(5):730-42.

21. Lewinsohn PM, Seeley JR, Roberts RE, et al. Center for Epidemiologic Studies Depression Scale (CES-D) as a screening instrument for depression among community-residing older adults. Psychol Aging. 1997;12(2):277-87.

22. Yesavage JA, Brink TL, Rose TL, et al. Development and validation of a geriatric depression screening scale: a preliminary report. J Psychiatr Res. 1982;17(1):37-49.

23. Ravens-Sieberer U, Wille N, Badia X, et al. Feasibility, reliability, and validity of the EQ-5D-Y: results from a multinational study. Qual Life Res. 2010;19(6):887-97. Epub 04/17.

24. Higgins JPT, Thomas J, Chandler J, et al. Cochrane handbook for systematic reviews of interventions, 2nd ed. Chichester, UK: John Wiley \& Sons; 2019.

25. Higgins JPT, Altman DG, Gøtzsche PC, et al. The Cochrane Collaboration's tool for assessing risk of bias in randomised trials. BMJ. 2011;343:d5928.

26. Akl EA, Sun X, Busse JW, et al. Specific instructions for estimating unclearly reported blinding status in randomized trials were reliable and valid. J Clin Epidemiol. 2012;65(3):262-67.

27. Guyatt GH, Oxman AD, Vist GE, et al. GRADE: an emerging consensus on rating quality of evidence and strength of recommendations. BMJ. 2008;336(7650):924-26.

28. Thorlund $\mathrm{K}$, Walter SD, Johnston $\mathrm{BC}$, et al. Pooling healthrelated quality of life outcomes in meta-analysis - a tutorial and review of methods for enhancing interpretability. Res Synthesis Meth. 2011;2(3):188-203.

29. Altman DG, Bland JM. Interaction revisited: the difference between two estimates. BMJ. 2003;326(7382):219.

30. Sterne JAC, Sutton AJ, Ioannidis JP, et al. Recommendations for examining and interpreting funnel plot asymmetry in metaanalyses of randomised controlled trials. BMJ. 2011;343:d4002.

31. Barlow JH, Turner AP, Gilchrist M. A randomised controlled trial of lay-led self-management for myocardial infarction patients who have completed cardiac rehabilitation. Eur $J$ Cardiovasc Nur. 2009;8(4):293-301.

32. Coull AJ, Taylor VH, Elton R, et al. A randomised controlled trial of senior Lay Health Mentoring in older people with ischaemic heart disease: The Braveheart Project. Age Ageing. 2004;33(4):348-54.

33. Parry MJ, Watt-Watson J, Hodnett E, et al. Cardiac home education and support trial (CHEST): a pilot study. Can J Cardiol. 2009;25(12):e393-e398.

34. Crotty M, Prendergast J, Battersby MW, et al. Self-management and peer support among people with arthritis on a hospital joint replacement waiting list: a randomised controlled trial. Osteoarthr Cartilage. 2009;17(11):1428-33.

35. Rantanen T, Äyräväinen I, Eronen J, et al. The effect of an outdoor activities' intervention delivered by older volunteers on the quality of life of older people with severe mobility limitations: a randomized controlled trial. Aging Clin Exp Res. 2015;27(2):161-69.

36. Robinson-Whelen $\mathrm{S}$, Hughes RB, Taylor HB, et al. Improving the health and health behaviors of women aging with physical disabilities: a peer-led health promotion program. Women's Health Issues. 2006;16(6):334-45.

37. Gagliardino JJ, Arrechea V, Assad D, et al. Type 2 diabetes patients educated by other patients perform at least as well as patients trained by professionals. Diabetes/Metabol Res Rev. 2013;29(2):152-60.

38. Johansson T, Keller S, Winkler $\mathrm{H}$, et al. Effectiveness of a Peer Support Programme versus Usual Care in Disease Management of Diabetes Mellitus Type 2 regarding Improvement of Metabolic Control: A Cluster-Randomised Controlled Trial. J Diabetes Res. 2016;2016:10.

39. Safford MM, Andreae S, Cherrington AL, et al. Peer coaches to improve diabetes outcomes in rural Alabama: a cluster randomized trial. Ann Fam Med. 2015;13(Suppl 1):S18-S26.

40. Crane-Okada R, Freeman E, Kiger H, et al. Senior peer counselling by telephone for psychosocial support after breast cancer surgery: effects at six months. Oncol Nurs Forum. 2012;39(1):78-89.

41. DeMello MM, Pinto BM, Mitchell S, Et Al. Peer support for physical activity adoption among breast cancer survivors: Do the helped resemble the helpers? Eur J Cancer Care. 2018;27(3): e12849. Epub 04/10.

42. Leone LA, Allicock M, Pignone MP, et al. cluster randomized trial of a church-based peer counselor and tailored newsletter intervention to promote colorectal cancer screening and physical activity among older African Americans. Health Educ Behav. 2016;43(5):568-76. Epub 10/29.

43. Weber BA, Roberts BL, Yarandi $\mathrm{H}$, et al. The impact of dyadic social support on self-efficacy and depression after radical prostatectomy. J Aging Health. 2007;19(4):630-45.

44. White V, Macvean M, Grogan S, et al. Can a tailored telephone intervention delivered by volunteers reduce the supportive care needs, anxiety and depression of people with colorectal cancer? A randomised controlled trial. Psychooncology. 2012;21(10):1053-62.

45. Buman MP, Giacobbi PR, Jr., Dzierzewski JM, et al. Peer volunteers improve long-term maintenance of physical activity with older adults: a randomized controlled trial. $J$ Phys Act Health. 2011;8(Suppl 2):S257-S266.

46. Castro CM, Pruitt LA, Buman MP, et al. Physical activity program delivery by professionals versus volunteers: the TEAM randomized trial. Health Psychol. 2011;30(3):285-94.

47. Chan AW, Yu DS, Choi KC. Effects of tai chi qigong on psychosocial well-being among hidden elderly, using elderly neighborhood volunteer approach: a pilot randomized controlled trial. Clin Interv Aging. 2017;12:85-96.

48. Charlesworth G, Shepstone L, Wilson E, et al. Does befriending by trained lay workers improve psychological well-being and quality of life for carers of people with dementia, and at what cost? A randomised controlled trial. Health Technol Assess. 2008;12(4).

49. McNeil JK. Effects of nonprofessional home visit programs for subclinically unhappy and unhealthy older adults. $J \mathrm{Appl}$ Gerontol. 1995;14(3):333-42.

50. Haider S, Dorner TE, Luger E, et al. Impact of a home-based physical and nutritional intervention program conducted by layvolunteers on handgrip strength in prefrail and frail older adults: a randomized control trial. PloS one. 2017;12(1):e0169613.

51. Kapan A, Winzer E, Haider S, et al. Impact of a lay-led homebased intervention programme on quality of life in communitydwelling pre-frail and frail older adults: a randomized controlled trial. BMC Geriatr. 2017;17(1):154. 


\section{MOORE: VOLUNTEER IMPACT ON SENIORS}

52. Allen RS, Harris GM, Burgio LD, et al. Can senior volunteers deliver reminiscence and creative activity interventions? Results of the legacy intervention family enactment randomized controlled trial. J Pain Symptom Manag. 2014;48(4):590-601. Epub 03/22.

53. Escolar Chua RL, de Guzman AB. Effects of third age learning programs on the life satisfaction, self-esteem, and depression level among a select group of community dwelling Filipino elderly. Educ Gerontol. 2014;40(2):77-90.

54. Iliffe S, Kendrick D, Morris R, et al. Multicentre cluster randomised trial comparing a community group exercise programme and home-based exercise with usual care for people aged 65 years and over in primary care. Health Technol Assess. 2014;18(49).

55. Mountain GA, Hind D, Gossage-Worrall R, et al. 'Putting Life in Years' (PLINY) telephone friendship groups research study: pilot randomised controlled trial. Trials. 2014;15(1):141.

56. Thomas GN, Macfarlane D, Guo B, et al. Health promotion in older Chinese: a 12-month cluster randomized controlled trial of pedometry and "peer support". Med Sci Sports Exercise. 2012;44(6):1157-66.

57. Hind D, Gossage-Worral R, Walters $\mathrm{S}$, et al. Putting Life in Years (PLINY): a randomised controlled trial and mixedmethods process evaluation of a telephone friendship intervention to improve mental well-being in independently living older people. Public Health Resh. 2014;2(7).

58. Kaczorowski J, Chambers LW, Dolovich L, et al. Improving cardiovascular health at population level: 39 community cluster randomised trial of Cardiovascular Health Awareness Program (CHAP). BMJ. 2011;342:d442.
59. Tang TS, Ayala GX, Cherrington A, et al. A review of volunteerbased peer support interventions in diabetes. Diabetes Spectrum. 2011;24(2):85-98.

60. Norris SL, Chowdhury FM, Van Le K, et al. Effectiveness of community health workers in the care of persons with diabetes. Diabetic Med. 2006;23(5):544-56.

61. Paterson DH, Warburton DE. Physical activity and functional limitations in older adults: a systematic review related to Canada's Physical Activity Guidelines. Int J Behav Nutr Phys Activity. 2010;7(1):1-22.

62. Cameron ID, Murray GR, Gillespie LD, et al. Interventions for preventing falls in older people in nursing care facilities and hospitals. Cochrane Db Syst Rev. 2010(1).

63. Gillespie LD, Robertson MC, Gillespie WJ, et al. Interventions for preventing falls in older people living in the community. Cochrane Db Syst Rev. 2012(9).

64. Langford CPH, Bowsher J, Maloney JP, et al. Social support: a conceptual analysis. $J$ Adv Nurs. 1997;25(1):95-100.

65. Bandura A, Cervone D. Self-evaluative and self-efficacy mechanisms governing the motivational effects of goal systems. $J$ Personality Soc Psychol. 1983;45(5):1017-28.

Correspondence to: Ainsley Moore, MD, CFPC, MSc, Department of Family Medicine, McMaster University, 100 Main St. W., 3rd Floor, Hamilton, ON L8P 1 H6

E-mail: amoore@mcmaster.ca 